\title{
Design, synthesis and antimicrobial activities of novel 1,3,5- thiadiazine-2-thione derivatives containing a 1,3,4-thiadiazole group
}

\author{
Jinghua Yan ${ }^{1}$, Weijie Si ${ }^{1,2}$, Haoran $\mathrm{Hu}^{1}$, Xu Zhao ${ }^{1}$, Min Chen ${ }^{\text {Corresp., }}{ }^{1}$, Xiaobin Wang ${ }^{1}$ \\ 1 Jiangsu Key Laboratory of Pesticide Science, College of Sciences, Nanjing Agricultural University, Nanjing, Jiangsu province, China \\ 2 Key Laboratory of Monitoring and Management of Crop Diseases and Pest Insects, Ministry of Agriculture, Nanjing Agricultural University, Nanjing, \\ Jiangsu province, China \\ Corresponding Author: Min Chen \\ Email address: chenmin@njau.edu.cn
}

A series of novel 1,3,5-thiadiazine-2-thione derivatives containing a 1,3,4-thiadiazole group was designed and synthesized. The structures of all the compounds were well characterized using ${ }^{1} \mathrm{H}$ NMR, ${ }^{13} \mathrm{C}$ NMR and HRMS, and further confirmed by the X-ray diffraction analysis of $\mathbf{8 d}$. The antimicrobial activities of all the target compounds against Xanthomonas oryzae pv. oryzicola (Xoc), Xanthomonas oryzae pv. oryzae (Xoo), Rhizoctonia solani $(R s)$ and Fusarium graminearum $(F g)$ were evaluated. The in vitro antimicrobial bioassays indicated that some title compounds exhibited noteworthy antimicrobial effects against the above strains. Notably, the compound $\mathrm{N}$-(5(ethylthio)-1,3,4-thiadiazol-2-yl)-2-(5-methyl-6-thioxo-1,3,5-thiadiazinan-3-yl)acetamide (8a) displayed obvious antibacterial effects against $X o c$ and $X o 0$ at $100 \mu \mathrm{g} / \mathrm{mL}$ with the inhibition rates of $30 \%$ and $56 \%$, respectively, which was better than the commercial bactericide thiodiazole-copper. In addition, the anti-Rs $\mathrm{EC}_{50}$ value of $8 \mathbf{a}$ was $33.70 \mu \mathrm{g} / \mathrm{mL}$, which was more effective than that of the commercial fungicide hymexazol $(67.10 \mu \mathrm{g} / \mathrm{mL})$. It was found that the substitutes in the 1,3,5-thiadiazine-2-thione and the 1,3,4-thiadiazole rings played a vital role in the antimicrobial activities of the title compounds. More active title compounds against phytopathogenic microorganisms might be obtained via the further structural modification. 
1 Design, synthesis and antimicrobial activities of novel

2 1,3,5-thiadiazine-2-thione derivatives containing a

3 1,3,4-thiadiazole group

\section{Jinghua Yan ${ }^{1}$, Weijie Si ${ }^{1,2}$, Haoran $\mathrm{Hu}^{1}$, Xu Zhao ${ }^{1}$, Min Chen ${ }^{1 *}$ and Xiaobin Wang1}

${ }^{1}$ Jiangsu Key Laboratory of Pesticide Science, College of Sciences, Nanjing Agricultural University, Nanjing 210095, China

${ }^{2}$ Key Laboratory of Monitoring and Management of Crop Diseases and Pest Insects, Ministry of Agriculture, Nanjing Agricultural University, Nanjing 210095, China

Corresponding Author:

Min Chen

No. 1, Weigang Road, Xuanwu District, Nanjing, Jiangsu, 210095, China

Email address: chenmin@njau.edu.cn

\section{Abstract}

A series of novel 1,3,5-thiadiazine-2-thione derivatives containing a 1,3,4-thiadiazole group was designed and synthesized. The structures of all the compounds were well characterized using ${ }^{1} \mathrm{H}$ NMR, ${ }^{13} \mathrm{C}$ NMR and HRMS, and further confirmed by the X-ray diffraction analysis of 8d. The antimicrobial activities of all the target compounds against Xanthomonas oryzae pv. oryzicola $(X o c)$, Xanthomonas oryzae pv. oryzae (Xoo), Rhizoctonia solani (Rs) and Fusarium graminearum $(F g)$ were evaluated. The in vitro antimicrobial bioassays indicated that some title compounds exhibited noteworthy antimicrobial effects against the above strains. Notably, the compound $N$-(5-(ethylthio)-1,3,4-thiadiazol-2-yl)-2-(5-methyl-6-thioxo-1,3,5-thiadiazinan-3yl)acetamide (8a) displayed obvious antibacterial effects against $X o c$ and $X o o$ at $100 \mu \mathrm{g} / \mathrm{mL}$ with the inhibition rates of $30 \%$ and $56 \%$, respectively, which was better than the commercial bactericide thiodiazole-copper. In addition, the anti- $R s \mathrm{EC}_{50}$ value of $\mathbf{8 a}$ was $33.70 \mu \mathrm{g} / \mathrm{mL}$, which was more effective than that of the commercial fungicide hymexazol $(67.10 \mu \mathrm{g} / \mathrm{mL})$. It was found that the substitutes in the 1,3,5-thiadiazine-2-thione and the 1,3,4-thiadiazole rings played a vital role in the antimicrobial activities of the title compounds. More active title compounds 
against phytopathogenic microorganisms might be obtained via the further structural modification.

Subjects Agricultural Science, Microbiology, Plant Science

Keywords 1,3,5-Thiadiazine-2-thione, 1,3,4-Thiadiazole, Crop protection, Antibacterial activity, Antifungal activity

\section{Introduction}

A variety of plant diseases, caused by pathogenic organisms, seriously affects the crop production, leading tremendous losses to agricultural economy every year (Wilson et al., 2009; Liu et al., 2013). Besides, the rapid emergence of resistant strains against traditional antimicrobial agents has become a huge challenge in the agricultural industry (Wang et al., 2013). In the last decades, researchers have found a large number of bioactive molecules with strong inhibitory effects on phytopathogenic bacteria and fungi. However, these compounds are rarely used in crop production due to the structural instability or poor control in farmland. Therefore, it is tardy to search highly-effective and eco-friendly agrochemicals for fighting against agricultural pathogenic microorganisms (Qian et al., 2010; Li et al., 2018). 1,3,5-Thiadiazine-2-thione derivatives were attractive bioactive molecules and exhibited antibacterial (Mao et al., 2017), antifungal (Vicentini et al., 2002), herbicidal (Vicentini et al., 2005), anticancer (El-Shorbagi et al., 2018), antileishmanial (Arshad et al., 2018), antiepileptic (Semreen et al., 2010), antimalarial (Coro et al., 2006), antioxidant (Ji et al., 2004), antitubercular (Katiyar et al., 2003) and trypanocidal (Coro et al., 2005) activities. Notably, the agricultural application of 1,3,5-thiadiazine-2-thione derivatives has also attracted great attention by chemists and biologists in the last three decades. For example, dazomet (Fig. 1A) and milneb (Fig. 1B), containg the 1,3,5-thiadiazine-2-thione moiety, were developed as the important agricultural nematicide and fungicide, respectively (Lam et al., 1993; Nakamura et al., 2010). Recently, Mao et al.(2017) found that dazomet could be applied as a promising agricultural bactericide to effectively control ginger blast in field trials (Mao et al., 2017). Meanwhile, our previous work found that 1,3,5-thiadiazine-2-thione derivatives with an acylhydrazine group displayed obvious antifungal activity in vitro and in vivo (Wang et al., 2018).

\section{1,3,4-thiadiazole derivatives attracted great attention by biochemists due to their various} bioactivities including antibacterial (Zhong et al., 2017), antifungal (Chen et al., 2000), insecticidal (Luo et al. 2007), antiviral (Chen et al., 2010), herbicidal (Cummings et al., 2009), anticancer (Casey et al., 2004), anti-tubercular (Foroumadi et al., 2003), antiparasitic (Coura et al., 2002), antidepressant (Siddiqui et al., 2011), antioxidant (Khan et al., 2010) and antiinflammatory (Kumar et al., 2008) activities. Among the above biological activities, the 
remarkable antimicrobial activity of 1,3,4-thiadiazole derivatives were well reported during the last decades. Thiodiazole-copper (Fig. 1C) and bismerthiazol (Fig. 1D), the representative agrochemicals containing the 1,3,4-thiadiazole group, were widely used to control bacterial diseases in crops. In addition, researchers found that the 1,3,4-thiadiazole derivatives bearing a sulfides moiety could effectively inhibit various agricultural bacteria (Wan et al., 2018).

On the basis of the above analysis, a series of 1,3,5-thiadiazine-2-thione derivatives, containing 1,3,4-thiadiazole scaffold (Fig. 2), were obtained to find novel antimicrobial candidates. The 1,3,4-thiadiazole fragment was introduced into the 5-position of the 1,3,5thiadiazine-2-thione according to the "combinatorial optimization" method (Fig. 3). The antibacterial activities against Xanthomonas oryzae pv. oryzae (Xoo) and Xanthomonas oryzae pv. oryzicola (Xoc) and the antifungal activity against Rhizoctonia solani (Rs) and Fusarium graminearum $(F g)$ were evaluated. Furthermore, the preliminary biological assay showed that some of the title compounds exhibited good antibacterial and antifungal activities. To the best of our knowledge, this is the first report about the synthesis and antimicrobial activity of 1,3,5thiadiazine-2-thione derivatives containing a 1,3,4-thiadiazole moiety.

\section{Materials and Methods}

\section{Materials}

All the solvents and reagents were purchased from commercial suppliers and used without further purification. The reaction was monitored by thin layer chromatography (TLC) on $\mathrm{GF}_{254}$ silica gel plates, which was visualized at UV $254 \mathrm{~nm}$. The melting points were measured with a SMP50 automatic melting point apparatus (Cole-Parmer, England). ${ }^{1} \mathrm{H}$ and ${ }^{13} \mathrm{C}$ NMR spectra were recorded on a BRUKER, AVANCE III $400 \mathrm{MHz}$ and probe of BBO spectrometer (Bruker Corporation, Germany) at room temperature with DMSO- $d_{6}$ as a solvent and TMS as an internal standard. High-resolution mass spectrometer (HRMS) data were measured on an AB SCIEX TripleTOF $5600^{+}$spectrometer (AB SCIEX, America) with the ESI charge source.

\section{General procedures for substituted 2-(6-thioxo-1,3,5-thiadiazinan-3-yl) acetic acids 3}

Carbon disulfide $(7.61 \mathrm{~g}, 100 \mathrm{mmol}$ ) was added dropwise to $100 \mathrm{~mL} 18 \%$ aqueous potassium hydroxide solution (18 $\mathrm{g}$ of potassium hydroxide dissolved in $82 \mathrm{~g}$ of water) containing phenylamine $(9.31 \mathrm{~g}, 100 \mathrm{mmol})$. After being stirred for $4 \mathrm{~h}$ at room temperature, the reaction mixture turned from colorless to orange and appeared white solid (compound 1a). Then, 37\% formaldehyde solution $(18.66 \mathrm{~g}, 230 \mathrm{mmol})$ was added into the reaction mixture, and the solution was stirred for another $1 \mathrm{~h}$ at room temperature. After being filtered, the filtrate was slowly dropped into a phosphate buffer solution $(\mathrm{pH} 7.8,100 \mathrm{~mL})$ containing glycine $(7.51 \mathrm{~g}, 100$ $\mathrm{mmol})$. After being stirred for $2 \mathrm{~h}$ at room temperature and being filtered, the filtrate was washed 
with diethyl ether until the color of organic phase was changed to colorless. The water phase was acidified with dilute hydrochloric acid to generate white precipitates, at $0-5{ }^{\circ} \mathrm{C}$. The precipitates were filtered, washed with iced ethanol, and dried to acquire the key intermediates 3a. This method was suitable for the synthesis of compounds $\mathbf{3 b}-\mathbf{3 d}$. The yields of compounds 3a-3d were between $46 \%$ and $71 \%$.

\section{General procedures for substituted 5-(ethylthio)-1,3,4-thiadiazol-2-amines 4}

Take the synthesis of compound $\mathbf{4 a}$ as an example: 5-amino-1,3,4-thiadiazole-2-thiol (2.0 g, 15 $\mathrm{mmol}$ ) and $\mathrm{K}_{2} \mathrm{CO}_{3}(2.76 \mathrm{~g}, 20 \mathrm{mmol}$ ) were dissolved in $20 \mathrm{~mL}$ DMF and stirred for 15 minutes at room temperature. The ethyl bromide $(2.18 \mathrm{~g}, 20 \mathrm{mmol})$ was added dropwise into the above mixture and stirred for $8 \mathrm{~h}$ at room temperature. Then the mixture was poured into cold water (10 $\mathrm{mL}$ ). The precipitated solid was filtered and recrystallized with the solvent of ethanol and water to gain pale yellow solid $\mathbf{4 a}$. The method was suitable for the synthesis of compounds $\mathbf{4 b}-\mathbf{4 d}$. The yields of compounds $\mathbf{4 a}-\mathbf{4 d}$ were between $77 \%$ and $81 \%$.

\section{General synthetic procedure for title compounds $5-8$.}

The intermediate 3a (0.50 g, $1.86 \mathrm{mmol}), O$-(Benzotriazole-1-yl)- $N, N, N^{\prime}, N^{\prime}$-tetramethyluronium tetrafluoroborate (TBTU, $0.72 \mathrm{~g}, 2.24 \mathrm{mmol})$, triethylamine $(0.38 \mathrm{~g}, 3.76 \mathrm{mmol})$ were added into dichloromethane $(30 \mathrm{~mL})$ and the mixture was stirred for $0.5 \mathrm{~h}$ at room temperature. Then, the intermediate $4 \mathbf{a}(0.45 \mathrm{~g}, 2.80 \mathrm{mmol})$ was added and stirred for another $2 \mathrm{~h}$ at room temperature. The resulting precipitate was filtered, washed with dichloromethane, and dried to give the desired product 5a. This method was suitable for the synthesis of title compounds 5-8 (Si et al., 2019). The yields of compounds 5-8 were between $41 \%$ and $79 \%$.

N-(5-(ethylthio)-1,3,4-thiadiazol-2-yl)-2-(5-phenyl-6-thioxo-1,3,5-thiadiazinan-3yl)acetamide (5a)

White solid, m.p. $158-160^{\circ} \mathrm{C}$, yield $61 \%$; ${ }^{1} \mathrm{H}$ NMR (400 MHz, DMSO- $\left.d_{6}\right) \delta 12.73(\mathrm{~s}, 1 \mathrm{H}, \mathrm{CONH})$, 7.47 (t, $J=7.6 \mathrm{~Hz}, 2 \mathrm{H}, \mathrm{PhH}), 7.35$ (t, $J=7.4 \mathrm{~Hz}, 1 \mathrm{H}, \mathrm{PhH}), 7.25$ (d, J=7.5 Hz, 2H, PhH), 4.76 (s, 2H, $\left.\mathrm{NCH}_{2} \mathrm{~N}\right), 4.69$ (s, 2H, $\left.\mathrm{SCH}_{2} \mathrm{~N}\right), 4.02$ (s, 2H, $\left.\mathrm{COCH}_{2}\right), 3.23$ (q, J=7.3 Hz, 2H, $\mathrm{CH}_{2} \mathrm{CH}_{3}$ ), $1.33\left(\mathrm{t}, J=7.3 \mathrm{~Hz}, 3 \mathrm{H}, \mathrm{CH}_{3}\right) ;{ }^{13} \mathrm{C} \mathrm{NMR}\left(101 \mathrm{MHz}, \mathrm{DMSO}-d_{6}\right) \delta 193.4,168.4,159.3,158.7$, 130.0, 128.3, 127.7, 74.1, 59.3, 53.2, 28.5, 15.2; HRMS (ESI) $m / z$ calcd for $\mathrm{C}_{15} \mathrm{H}_{18} \mathrm{~N}_{5} \mathrm{OS}_{4}$ $\left([\mathrm{M}+\mathrm{H}]^{+}\right): 412.0388$, found: 412.0388 .

\section{$N$-(5-(benzylthio)-1,3,4-thiadiazol-2-yl)-2-(5-phenyl-6-thioxo-1,3,5-thiadiazinan-3-} yl)acetamide (5b)

White solid, m.p. $175-176^{\circ} \mathrm{C}$, yield $66 \% ;{ }^{1} \mathrm{H}$ NMR (400 MHz, DMSO- $\left.d_{6}\right) \delta 7.46(\mathrm{t}, J=7.7 \mathrm{~Hz}$, 
2H, $\mathrm{PhH}), 7.40$ (d, $J=7.3 \mathrm{~Hz}, 2 \mathrm{H}, \mathrm{PhH}), 7.34$ (dd, $J=17.5,7.6 \mathrm{~Hz}, 3 \mathrm{H}, \mathrm{PhH}), 7.28$ (d, $J=7.1$ $\mathrm{Hz}, 1 \mathrm{H}, \mathrm{PhH}), 7.24(\mathrm{~d}, J=7.6 \mathrm{~Hz}, 2 \mathrm{H}, \mathrm{PhH}), 4.75\left(\mathrm{~s}, 2 \mathrm{H}, \mathrm{NCH}_{2} \mathrm{~N}\right), 4.68\left(\mathrm{~s}, 2 \mathrm{H}, \mathrm{SCH}_{2} \mathrm{~N}\right), 4.48(\mathrm{~s}$, $\left.2 \mathrm{H}, \mathrm{SCH}_{2} \mathrm{Ph}\right), 4.00\left(\mathrm{~s}, 2 \mathrm{H}, \mathrm{COCH}_{2}\right) ;{ }^{13} \mathrm{C} \mathrm{NMR}\left(101 \mathrm{MHz}, \mathrm{DMSO}-d_{6}\right) \delta 193.4,168.4,159.1,158.7$, 144.7, 137.2, 129.9, 129.5, 129.0, 128.3, 128.1, 127.7, 74.1, 59.3, 53.2, 38.0; HRMS (ESI) $\mathrm{m} / \mathrm{z}$ calcd for $\mathrm{C}_{20} \mathrm{H}_{19} \mathrm{~N}_{5} \mathrm{NaOS}_{4}\left([\mathrm{M}+\mathrm{Na}]^{+}\right)$: 496.0365 , found: 496.0366 .

\section{$N$-(5-((4-methylbenzyl)thio)-1,3,4-thiadiazol-2-yl)-2-(5-phenyl-6-thioxo-1,3,5-thiadiazinan-} 3-yl)acetamide (5c)

White solid, m.p. $169-171^{\circ} \mathrm{C}$, yield $67 \%$; ${ }^{1} \mathrm{H}$ NMR (400 MHz, DMSO- $\left.d_{6}\right) \delta 7.46$ (t, $J=7.2 \mathrm{~Hz}$, 2H, PhH), 7.38-7.32 (m, 1H, PhH), 7.26 (dd, J=14.8, 7.7 Hz, 4H, PhH), 7.13 (d, J=7.5 Hz, 2H, $\mathrm{PhH}), 4.75\left(\mathrm{~s}, 2 \mathrm{H}, \mathrm{NCH}_{2} \mathrm{~N}\right), 4.68\left(\mathrm{~s}, 2 \mathrm{H}, \mathrm{SCH}_{2} \mathrm{~N}\right), 4.44\left(\mathrm{~s}, 2 \mathrm{H}, \mathrm{SCH}_{2} \mathrm{Ph}\right), 4.00\left(\mathrm{~s}, 2 \mathrm{H}, \mathrm{COCH}_{2}\right)$, $2.27\left(\mathrm{~s}, 3 \mathrm{H}, \mathrm{CH}_{3}\right) ;{ }^{13} \mathrm{C}$ NMR (101 MHz, DMSO- $\left.d_{6}\right) \delta 193.4,168.4,159.1,158.8,144.7,137.3$, 134.0, 129.9, 129.6, 129.4, 128.3, 127.7, 74.1, 59.3, 53.2, 37.8, 21.2; HRMS (ESI) $\mathrm{m} / \mathrm{z}$ calcd for $\mathrm{C}_{21} \mathrm{H}_{22} \mathrm{~N}_{5} \mathrm{OS}_{4}\left([\mathrm{M}+\mathrm{H}]^{+}\right)$: 488.0702, found: 488.0696 .

$\mathrm{N}$-(5-((4-chlorobenzyl)thio)-1,3,4-thiadiazol-2-yl)-2-(5-phenyl-6-thioxo-1,3,5-thiadiazinan-3yl)acetamide (5d)

White solid, m.p. $179-181^{\circ} \mathrm{C}$, yield $69 \%$; ${ }^{1} \mathrm{H}$ NMR (400 MHz, DMSO- $\left.d_{6}\right) \delta 7.49-7.32(\mathrm{~m}, 7 \mathrm{H}$, $\mathrm{PhH}), 7.24(\mathrm{~d}, J=7.5 \mathrm{~Hz}, 2 \mathrm{H}, \mathrm{PhH}), 4.75\left(\mathrm{~s}, 2 \mathrm{H}, \mathrm{NCH}_{2} \mathrm{~N}\right), 4.68\left(\mathrm{~s}, 2 \mathrm{H}, \mathrm{SCH}_{2} \mathrm{~N}\right), 4.49(\mathrm{~d}, J=$ $\left.11.2 \mathrm{~Hz}, 2 \mathrm{H}, \mathrm{SCH}_{2} \mathrm{Ph}\right), 4.00\left(\mathrm{~s}, 2 \mathrm{H}, \mathrm{COCH}_{2}\right) ;{ }^{13} \mathrm{C} \mathrm{NMR}\left(101 \mathrm{MHz}, \mathrm{DMSO}-d_{6}\right) \delta 193.4,168.5$, 159.3, 158.3, 144.7, 136.5, 132.6, 131.3, 129.9, 129.0, 128.3, 127.7, 74.1, 59.3, 53.2, 37.1; HRMS (ESI) $\mathrm{m} / z$ calcd for $\mathrm{C}_{20} \mathrm{H}_{18} \mathrm{ClN}_{5} \mathrm{NaOS}_{4}\left([\mathrm{M}+\mathrm{Na}]^{+}\right): 529.9975$, found: 529.9970 .

\section{2-(5-benzyl-6-thioxo-1,3,5-thiadiazinan-3-yl)- $N$-(5-(ethylthio)-1,3,4-thiadiazol-2-} yl)acetamide (6a)

White solid, m.p. $179-181^{\circ} \mathrm{C}$, yield $79 \% ;{ }^{1} \mathrm{H}$ NMR (400 MHz, DMSO- $\left.d_{6}\right) \delta 12.50$ (s, $\left.1 \mathrm{H}, \mathrm{CONH}\right)$, 7.35 (d, $J=7.5 \mathrm{~Hz}, 2 \mathrm{H}, \mathrm{PhH}), 7.27$ (t, $J=7.6 \mathrm{~Hz}, 2 \mathrm{H}, \mathrm{PhH}), 7.12(\mathrm{t}, J=7.3 \mathrm{~Hz}, 1 \mathrm{H}, \mathrm{PhH}), 5.28$ $\left(\mathrm{s}, 2 \mathrm{H}, \mathrm{NCH}_{2} \mathrm{Ph}\right), 4.58\left(\mathrm{~s}, 2 \mathrm{H}, \mathrm{NCH}_{2} \mathrm{~N}\right), 4.50\left(\mathrm{~s}, 2 \mathrm{H}, \mathrm{SCH}_{2} \mathrm{~N}\right), 3.69\left(\mathrm{~s}, 2 \mathrm{H}, \mathrm{COCH}_{2}\right), 3.22(\mathrm{q}, J=$ $\left.7.3 \mathrm{~Hz}, 2 \mathrm{H}, \mathrm{CH}_{2} \mathrm{CH}_{3}\right), 1.34\left(\mathrm{t}, J=7.3 \mathrm{~Hz}, 3 \mathrm{H}, \mathrm{CH}_{3}\right) ;{ }^{13} \mathrm{C}$ NMR $\left(101 \mathrm{MHz}\right.$, DMSO- $\left.d_{6}\right) \delta 192.2$, $167.8,159.1,158.6,136.0,129.0,128.4,127.9,68.9,59.3,53.3,52.7,28.6,15.2$; HRMS (ESI) $\mathrm{m} / z$ calcd for $\mathrm{C}_{16} \mathrm{H}_{20} \mathrm{~N}_{5} \mathrm{OS}_{4}\left([\mathrm{M}+\mathrm{H}]^{+}\right): 426.0545$, found: 426.0542 .

\section{2-(5-benzyl-6-thioxo-1,3,5-thiadiazinan-3-yl)- $N$-(5-(benzylthio)-1,3,4-thiadiazol-2-} yl)acetamide (6b)

White solid, m.p. $160-162^{\circ} \mathrm{C}$, yield $79 \% ;{ }^{1} \mathrm{H}$ NMR (400 MHz, DMSO- $\left.d_{6}\right) \delta 12.46$ (s, $1 \mathrm{H}, \mathrm{CONH}$ ), 7.41 (d, $J=7.4 \mathrm{~Hz}, 2 \mathrm{H}, \mathrm{PhH}), 7.33$ (s, 4H, PhH), 7.30-7.21 (m, 3H, PhH), 7.08 (t, $J=7.3 \mathrm{~Hz}$, 
177

178

179

180

181

182

183

184

185

186

187

188

189

190

191

192

193

194

195

196

197

198

199

200

201

202

203

204

205

206

207

208

209

210

211

212

1H, $\mathrm{PhH}$ ), 5.27 (s, $2 \mathrm{H}, \mathrm{NCH}_{2} \mathrm{Ph}$ ), 4.57 (s, $2 \mathrm{H}, \mathrm{SCH}_{2} \mathrm{Ph}$ ), 4.48 (s, 4H, $\mathrm{NCH}_{2} \mathrm{NCH}_{2} \mathrm{~S}$ ), 3.66 (s, $2 \mathrm{H}$, $\left.\mathrm{COCH}_{2}\right) ;{ }^{13} \mathrm{C}$ NMR $\left(101 \mathrm{MHz}\right.$, DMSO- $\left.d_{6}\right) \delta 192.2,167.9,159.0,158.4,137.2,136.0,129.4$, 129.0, 128.4, 128.1, 127.9, 68.89, 59.3, 53.4, 52.7, 38.0; HRMS (ESI) $\mathrm{m} / \mathrm{z}$ calcd for $\mathrm{C}_{21} \mathrm{H}_{22} \mathrm{~N}_{5} \mathrm{OS}_{4}\left([\mathrm{M}+\mathrm{H}]^{+}\right): 488.0702$, found: 488.0695 .

2-(5-benzyl-6-thioxo-1,3,5-thiadiazinan-3-yl)- $N$-(5-((4-methylbenzyl)thio)-1,3,4-thiadiazol2-yl)acetamide (6c)

White solid, m.p. $179-181{ }^{\circ} \mathrm{C}$, yield $71 \%$; ${ }^{1} \mathrm{H}$ NMR (400 MHz, DMSO- $\left.d_{6}\right) \delta 12.43$ (s, $1 \mathrm{H}, \mathrm{CONH}$ ), $7.34(\mathrm{~d}, J=7.4 \mathrm{~Hz}, 2 \mathrm{H}, \mathrm{PhH}), 7.31-7.21(\mathrm{~m}, 4 \mathrm{H}, \mathrm{PhH}), 7.14(\mathrm{~d}, J=7.2 \mathrm{~Hz}, 2 \mathrm{H}, \mathrm{PhH}), 7.09$ (t, $J$ $=7.0 \mathrm{~Hz}, 1 \mathrm{H}, \mathrm{PhH}), 5.28\left(\mathrm{~s}, 2 \mathrm{H}, \mathrm{NCH}_{2} \mathrm{Ph}\right), 4.57\left(\mathrm{~s}, 2 \mathrm{H}, \mathrm{SCH}_{2} \mathrm{Ph}\right), 4.48\left(\mathrm{~s}, 2 \mathrm{H}, \mathrm{NCH}_{2} \mathrm{~N}\right), 4.44(\mathrm{~s}$, $\left.2 \mathrm{H}, \mathrm{SCH}_{2} \mathrm{~N}\right), 3.66\left(\mathrm{~s}, 2 \mathrm{H}, \mathrm{COCH}_{2}\right), 2.27\left(\mathrm{~s}, 3 \mathrm{H}, \mathrm{CH}_{3}\right) ;{ }^{13} \mathrm{C} \mathrm{NMR}\left(101 \mathrm{MHz}, \mathrm{DMSO}-d_{6}\right) \delta 192.2$, 167.9, 156.0, 158.6, 137.3, 136.0, 134.1, 129.6, 129.4, 129.0, 128.5, 127.9, 68.9, 59.3, 53.4, 52.7, 37.9, 21.2; HRMS (ESI) $m / z$ calcd for $\mathrm{C}_{22} \mathrm{H}_{24} \mathrm{~N}_{5} \mathrm{OS}{ }_{4}\left([\mathrm{M}+\mathrm{H}]^{+}\right): 502.0858$, found: 502.0850 .

2-(5-benzyl-6-thioxo-1,3,5-thiadiazinan-3-yl)- $\mathrm{N}$-(5-((4-chlorobenzyl)thio)-1,3,4-thiadiazol-2yl)acetamide (6d)

White solid, m.p. $175-177^{\circ} \mathrm{C}$, yield $72 \%$; ${ }^{1} \mathrm{H}$ NMR $\left(400 \mathrm{MHz}, \mathrm{DMSO}-d_{6}\right) \delta 7.41(\mathrm{dd}, J=16.2$, $7.8 \mathrm{~Hz}, 4 \mathrm{H}, \mathrm{PhH}), 7.34$ (d, $J=7.4 \mathrm{~Hz}, 2 \mathrm{H}, \mathrm{PhH}), 7.24$ (t, $J=7.3 \mathrm{~Hz}, 2 \mathrm{H}, \mathrm{PhH}), 7.07$ (t, $J=7.3$ $\mathrm{Hz}, 1 \mathrm{H}, \mathrm{PhH}), 5.28\left(\mathrm{~s}, 2 \mathrm{H}, \mathrm{NCH}_{2} \mathrm{Ph}\right), 4.57$ (s, 2H, $\left.\mathrm{SCH}_{2} \mathrm{Ph}\right), 4.48$ (s, $\left.4 \mathrm{H}, \mathrm{NCH}_{2} \mathrm{NCH}_{2} \mathrm{~S}\right), 3.66$ (s, $\left.2 \mathrm{H}, \mathrm{COCH}_{2}\right) ;{ }^{13} \mathrm{C}$ NMR $\left(101 \mathrm{MHz}, \mathrm{DMSO}-d_{6}\right) \delta 192.12,167.9,159.2,158.0,136.5,136.0,132.6$, $131.33,129.0,129.0,128.4,127.9,68.9,59.3,53.4,52.7,37.2$; HRMS (ESI) $m / z$ calcd for $\mathrm{C}_{21} \mathrm{H}_{20} \mathrm{ClN}_{5} \mathrm{NaOS}_{4}\left([\mathrm{M}+\mathrm{Na}]^{+}\right): 544.0132$, found: 544.0125 .

\section{$N$-(5-(ethylthio)-1,3,4-thiadiazol-2-yl)-2-(5-(4-fluorophenyl)-6-thioxo-1,3,5-thiadiazinan-3-} yl)acetamide (7a)

White solid, m.p. $173-175^{\circ} \mathrm{C}$, yield $41 \%$; ${ }^{1} \mathrm{H}$ NMR (400 MHz, DMSO- $\left.d_{6}\right) \delta 7.30(\mathrm{~d}, \mathrm{~J}=6.9 \mathrm{~Hz}$, $4 \mathrm{H}, \mathrm{PhH}), 4.76\left(\mathrm{~s}, 2 \mathrm{H}, \mathrm{NCH}_{2} \mathrm{~N}\right), 4.69\left(\mathrm{~s}, 2 \mathrm{H}, \mathrm{SCH}_{2} \mathrm{~N}\right), 4.01\left(\mathrm{~s}, 2 \mathrm{H}, \mathrm{COCH}_{2}\right), 3.22$ (q, J = $7.3 \mathrm{~Hz}$, $\left.2 \mathrm{H}, \mathrm{CH}_{2} \mathrm{CH}_{3}\right), 1.33\left(\mathrm{t}, \mathrm{J}=7.3 \mathrm{~Hz}, 3 \mathrm{H}, \mathrm{CH}_{3}\right) ;{ }^{13} \mathrm{C}$ NMR $\left(101 \mathrm{MHz}\right.$, DMSO- $\left.d_{6}\right) \delta$ 194.0, 168.4, 159.3, 158.7, 140.8, 130.0, 129.9, 116.9, 116.6, 74.1, 59.3, 53.2, 28.5, 15.2; HRMS (ESI) $\mathrm{m} / \mathrm{z}$ calcd for $\mathrm{C}_{16} \mathrm{H}_{19} \mathrm{FN}_{5} \mathrm{OS}_{4}\left([\mathrm{M}+\mathrm{H}]^{+}\right)$: 430.0295 , found: 430.0291 .

$N$-(5-(benzylthio)-1,3,4-thiadiazol-2-yl)-2-(5-(4-fluorophenyl)-6-thioxo-1,3,5-thiadiazinan-3yl)acetamide (7b)

White solid, m.p. $163-165^{\circ} \mathrm{C}$, yield 57\%; ${ }^{1} \mathrm{H}$ NMR (400 MHz, DMSO- $\left.d_{6}\right) \delta 12.73$ (s, $1 \mathrm{H}, \mathrm{CONH}$ ), $7.44(\mathrm{~d}, J=7.0 \mathrm{~Hz}, 2 \mathrm{H}, \mathrm{PhH}), 7.40-7.30(\mathrm{~m}, 7 \mathrm{H}, \mathrm{PhH}), 4.79\left(\mathrm{~s}, 2 \mathrm{H}, \mathrm{NCH}_{2} \mathrm{~N}\right), 4.72(\mathrm{~s}, 2 \mathrm{H}$, $\left.\mathrm{SCH}_{2} \mathrm{~N}\right), 4.53\left(\mathrm{~s}, 2 \mathrm{H}, \mathrm{SCH}_{2} \mathrm{Ph}\right), 4.05\left(\mathrm{~s}, 2 \mathrm{H}, \mathrm{COCH}_{2}\right) ;{ }^{13} \mathrm{C} \mathrm{NMR}\left(101 \mathrm{MHz}, \mathrm{DMSO}-d_{6}\right) \delta$ 194.0, 
213

214

215

216

217

218

219

220

221

222

223

224

225

226

227

228

229

230

231

232

233

234

235

236

237

238

239

240

241

242

243

244

245

246

247

248

$168.4,159.1,158.8,140.8,137.2,130.0,129.9,129.5,129.0,128.1,116.9,116.6,74.1,59.4$, 53.2, 38.0; HRMS (ESI) $\mathrm{m} / z$ calcd for $\mathrm{C}_{20} \mathrm{H}_{19} \mathrm{FN}_{5} \mathrm{OS}_{4}\left([\mathrm{M}+\mathrm{H}]^{+}\right)$: 492.0451 , found: 492.0444 .

2-(5-(4-fluorophenyl)-6-thioxo-1,3,5-thiadiazinan-3-yl)- $N$-(5-((4-methylbenzyl)thio)-1,3,4thiadiazol-2-yl)acetamide (7c)

White solid, m.p. $170-171{ }^{\circ} \mathrm{C}$, yield $49 \%$; ${ }^{1} \mathrm{H}$ NMR (400 MHz, DMSO- $\left.d_{6}\right) \delta 7.29(\mathrm{t}, J=7.8 \mathrm{~Hz}$, $6 \mathrm{H}, \mathrm{PhH}), 7.13$ (d, J=7.6 Hz, 2H, PhH), $4.75\left(\mathrm{~s}, 2 \mathrm{H}, \mathrm{NCH}_{2} \mathrm{~N}\right), 4.68\left(\mathrm{~s}, 2 \mathrm{H}, \mathrm{SCH}_{2} \mathrm{~N}\right), 4.44(\mathrm{~s}, 2 \mathrm{H}$, $\left.\mathrm{SCH}_{2} \mathrm{Ph}\right), 4.00\left(\mathrm{~s}, 2 \mathrm{H}, \mathrm{COCH}_{2}\right), 2.27\left(\mathrm{~s}, 3 \mathrm{H}, \mathrm{CH}_{3}\right) ;{ }^{13} \mathrm{C} \mathrm{NMR}\left(101 \mathrm{MHz}, \mathrm{DMSO}-d_{6}\right) \delta 194.0$, $168.5,159.1,158.8,140.8,137.3,134.0,130.0,129.9,129.6,129.4,116.9,116.6,74.1,59.4$, 53.3, 37.8, 21.2; HRMS (ESI) $m / z$ calcd for $\mathrm{C}_{21} \mathrm{H}_{21} \mathrm{FN}_{5} \mathrm{OS}_{4}\left([\mathrm{M}+\mathrm{H}]^{+}\right)$: 506.0608, found: 506.0598 .

\section{$\mathrm{N}$-(5-((4-chlorobenzyl)thio)-1,3,4-thiadiazol-2-yl)-2-(5-(4-fluorophenyl)-6-thioxo-1,3,5-} thiadiazinan-3-yl)acetamide (7d)

White solid, m.p. $174-175^{\circ} \mathrm{C}$, yield $48 \%$; ${ }^{1} \mathrm{H}$ NMR (400 MHz, DMSO- $\left.d_{6}\right) \delta 7.40$ (dd, $J=16.1$, $7.9 \mathrm{~Hz}, 4 \mathrm{H}, \mathrm{PhH}), 7.30$ (d, J=6.5 Hz, 4H, PhH), 4.75 (s, 2H, $\left.\mathrm{NCH}_{2} \mathrm{~N}\right), 4.68\left(\mathrm{~s}, 2 \mathrm{H}, \mathrm{SCH}_{2} \mathrm{~N}\right)$, $4.48\left(\mathrm{~s}, 2 \mathrm{H}, \mathrm{SCH}_{2} \mathrm{Ph}\right), 4.00\left(\mathrm{~s}, 2 \mathrm{H}, \mathrm{COCH}_{2}\right) ;{ }^{13} \mathrm{C} \mathrm{NMR}\left(101 \mathrm{MHz}, \mathrm{DMSO}-d_{6}\right) \delta 194.0,168.5$, 159.3, 158.3, 140.8, 136.5, 132.6, 131.3, 130.0, 129.9, 129.0, 116.9, 116.6, 74.1, 59.4, 53.3, 37.1; HRMS (ESI) $m / z$ calcd for $\mathrm{C}_{20} \mathrm{H}_{18} \mathrm{ClFN}_{5} \mathrm{OS}_{4}\left([\mathrm{M}+\mathrm{H}]^{+}\right)$: 526.0061 , found: 526.0049 .

\section{$N$-(5-(ethylthio)-1,3,4-thiadiazol-2-yl)-2-(5-methyl-6-thioxo-1,3,5-thiadiazinan-3-} yl)acetamide (8a)

White solid, m.p. $159-161^{\circ} \mathrm{C}$, yield $45 \%$; ${ }^{1} \mathrm{H}$ NMR $\left(400 \mathrm{MHz}, \mathrm{DMSO}-d_{6}\right) \delta 4.55(\mathrm{~s}, 4 \mathrm{H}$, $\left.\mathrm{NCH}_{2} \mathrm{NCH}_{2} \mathrm{~S}\right), 3.83\left(\mathrm{~s}, 2 \mathrm{H}, \mathrm{COCH}_{2}\right), 3.37\left(\mathrm{~s}, 3 \mathrm{H}, \mathrm{NCH}_{3}\right), 3.23\left(\mathrm{q}, \mathrm{J}=7.3 \mathrm{~Hz}, 2 \mathrm{H}, \mathrm{CH}_{2} \mathrm{CH}_{3}\right)$, 1.35 (t, J = 7.3 Hz, 3H, $\left.\mathrm{CH}_{2} \mathrm{CH}_{3}\right) ;{ }^{13} \mathrm{C}$ NMR (101 MHz, DMSO- $\left.d_{6}\right) \delta 190.5,168.5,159.2,158.8$, 71.8, 59.3, 58.8, 53.3, 28.6, 15.2; HRMS (ESI) $m / z$ calcd for $\mathrm{C}_{10} \mathrm{H}_{16} \mathrm{~N}_{5} \mathrm{OS}_{4}\left([\mathrm{M}+\mathrm{H}]^{+}\right): 350.0232$, found: 350.0230 .

\section{N-(5-(benzylthio)-1,3,4-thiadiazol-2-yl)-2-(5-methyl-6-thioxo-1,3,5-thiadiazinan-3-} yl)acetamide (8b)

White solid, m.p.166-168 ${ }^{\circ} \mathrm{C}$, yield 66\%; ${ }^{1} \mathrm{H}$ NMR (400MHz, DMSO- $\left.d_{6}\right) \delta 12.45(\mathrm{~s}, 1 \mathrm{H}, \mathrm{CONH})$, 7.41 (d, J = 7.4 Hz, 2H, PhH), 7.34 (t, J = 7.3 Hz, 2H, PhH), 7.31-7.24 (m, 1H, PhH), 4.54 (s, 4H, $\left.\mathrm{NCH}_{2} \mathrm{NCH}_{2} \mathrm{~S}\right), 4.49\left(\mathrm{~s}, 2 \mathrm{H}, \mathrm{SCH}_{2} \mathrm{Ph}\right), 3.82\left(\mathrm{~s}, 2 \mathrm{H}, \mathrm{COCH}_{2}\right), 3.37\left(\mathrm{~s}, 3 \mathrm{H}, \mathrm{NCH}_{3}\right) ;{ }^{13} \mathrm{C} \mathrm{NMR}(101$ MHz, DMSO- $\left.d_{6}\right) \delta 190.5,168.6,159.2,158.6,137.2,129.5,129.0,128.1,71.8,58.8,53.3,38.0$; HRMS (ESI) $m / z$ calcd for $\mathrm{C}_{15} \mathrm{H}_{17} \mathrm{~N}_{5} \mathrm{NaOS}_{4}\left([\mathrm{M}+\mathrm{Na}]^{+}\right)$: 434.0208, found: 434.0204 . 
2-(5-methyl-6-thioxo-1,3,5-thiadiazinan-3-yl)- $N$-(5-((4-methylbenzyl)thio)-1,3,4-thiadiazol2-yl)acetamide (8c)

White solid, m.p. $168-170^{\circ} \mathrm{C}$, yield $56 \%$; ${ }^{1} \mathrm{H}$ NMR $\left(400 \mathrm{MHz}, \mathrm{DMSO}-d_{6}\right) \delta 7.28(\mathrm{~d}, J=7.5 \mathrm{~Hz}$, $2 \mathrm{H}, \mathrm{PhH}$ ), 7.13 (d, J=7.7 Hz, 2H, $\mathrm{PhH}$ ), 4.53 (s, 4H, $\mathrm{NCH}_{2} \mathrm{NCH}_{2} \mathrm{~S}$ ), 4.44 (s, $2 \mathrm{H}, \mathrm{SCH}_{2} \mathrm{Ph}$ ), 3.81 (s, $\left.2 \mathrm{H}, \mathrm{COCH}_{2}\right), 3.36\left(\mathrm{~s}, 3 \mathrm{H}, \mathrm{NCH}_{3}\right), 2.27\left(\mathrm{~s}, 3 \mathrm{H}, \mathrm{PhCH}_{3}\right) ;{ }^{13} \mathrm{C} \mathrm{NMR}\left(101 \mathrm{MHz}, \mathrm{DMSO}-d_{6}\right) \delta$ 190.5, 168.6, 159.2, 158.8, 137.3, 134.0, 129.6, 129.4, 71.8, 58.8, 53.3, 37.8, 21.2; HRMS (ESI) $\mathrm{m} / z$ calcd for $\mathrm{C}_{16} \mathrm{H}_{20} \mathrm{~N}_{5} \mathrm{OS}_{4}\left([\mathrm{M}+\mathrm{H}]^{+}\right): 426.0545$, found: 426.0541 .

$N$-(5-((4-chlorobenzyl)thio)-1,3,4-thiadiazol-2-yl)-2-(5-methyl-6-thioxo-1,3,5-thiadiazinan-3yl)acetamide (8d)

White solid, m.p. $161-163^{\circ} \mathrm{C}$, yield $56 \%$; ${ }^{1} \mathrm{H}$ NMR (400 MHz, DMSO- $\left.d_{6}\right) \delta 12.49$ (s, $1 \mathrm{H}, \mathrm{CONH}$ ), 7.41 (q, $J=8.1 \mathrm{~Hz}, 4 \mathrm{H}, \mathrm{PhH}), 4.53$ (s, $\left.4 \mathrm{H}, \mathrm{NCH}_{2} \mathrm{NCH}_{2} \mathrm{~S}\right), 4.48\left(\mathrm{~s}, 2 \mathrm{H}, \mathrm{SCH}_{2} \mathrm{Ph}\right), 3.81(\mathrm{~s}, 2 \mathrm{H}$, $\mathrm{COCH}_{2}$ ), $3.36\left(\mathrm{~s}, 3 \mathrm{H}, \mathrm{CH}_{3}\right) ;{ }^{13} \mathrm{C}$ NMR (101 MHz, DMSO- $\left.d_{6}\right) \delta 190.5,168.7,159.5,158.2,136.5$, 132.6, 131.3, 129.0, 71.8, 58.8, 53.4, 37.1; HRMS (ESI) $\mathrm{m} / z$ calcd for $\mathrm{C}_{15} \mathrm{H}_{16} \mathrm{ClN}_{5} \mathrm{NaOS}_{4}$ $\left([\mathrm{M}+\mathrm{Na}]^{+}\right): 467.9819$, found: 467.9814 .

\section{Crystal structure determination}

The title compound $\mathbf{8 d}$ was recrystallized from a mixture of DMF and methanol (V: V=1:1) to obtain a suitable single crystal. The X-ray single crystal diffraction data was collected on an Agilent Super Nova (Dual, Cu at zero, AtlasS2) single crystal diffractometer at 100.00 (10) K with the monochromatized MoK $\alpha$ radiation $(\lambda=0.71073 \AA)$ using $w$ scan mode. The CrysAlisPro program was used to integrate the diffraction profile. The structure was solved directly and optimized by using full matrix least square method via SHELXL (Sheldrick, 1997). All the non-hydrogen atoms were refined by full-matrix least-squares technique on $F^{2}$ with anisotropic thermal parameters. All the hydrogen atoms were positioned geometrically and refined using a riding model. PLATON program was used for the structural analysis and the diamond program was used for the drawings (Spek, 2003).

\section{Antibacterial activities test in vitro}

The antibacterial activities of title compounds against Xoo and Xoc were evaluated by the turbidimeter test (Li et al., 2014; Wang et al., 2013; Xu et al., 2012). The compounds were dissolved in dimethylsulfoxide (DMSO) and diluted with water (containing $0.1 \%$ Tween-20) to obtain a solution with a final concentration of 100 and $50 \mu \mathrm{g} / \mathrm{mL}$ by adding different amounts water. DMSO in sterile distilled water served as a blank control, thiodiazole-copper served as positive control. Approximately $1 \mathrm{~mL}$ of sample liquid was added to the nontoxic nutrient broth (NB, $3.0 \mathrm{~g}$ of beef extract, $5.0 \mathrm{~g}$ of peptone, $1.0 \mathrm{~g}$ of yeast powder, $10.0 \mathrm{~g}$ of glucose, and 1000 
$\mathrm{mL}$ of distilled water, $\mathrm{pH} 7.0$ to 7.2 ) liquid medium in $4 \mathrm{~mL}$ tubes. Then, about $40 \mu \mathrm{L}$ of solvent $\mathrm{NB}$ containing Xoo or Xoc was added to $5 \mathrm{~mL}$ of solvent NB containing the test compounds and positive control. The inoculated test tubes were incubated at $28 \pm 1{ }^{\circ} \mathrm{C}$ and continuously cultured shakily at $180 \mathrm{rpm}$ for 2-3 days. The growth of the cultures was monitored on a microplate reader by measuring the optical density at $600 \mathrm{~nm}\left(\mathrm{OD}_{600}\right)$ given by turbidity ${ }_{\text {corrected values }}=$ $\mathrm{OD}_{\text {bacterium }}-\mathrm{OD}_{\text {no bacterium, }}$ and then the inhibition rate $I(\%)$ was calculated by $I=\left(C_{\text {tur }}-T_{\text {tur }}\right) / C_{\text {tur }}$ $\times 100 \% . C_{\text {tur }}$ is the corrected turbidity values of bacterial growth on untreated NB (blank control), and $T_{\text {tur }}$ is the corrected turbidity values of bacterial growth on treated NB.

\section{Antifungal activities test in vitro}

$R s$ and $F g$, which were the representative plant pathogenic fungi, were chosen as the test strains. The antifungal activities of title compounds against $R s$ and $F g$ in vitro were tested by the mycelium growth rate method (Wang et al., 2017; Chen et al., 2012). Different doses of compounds were dissolved in DMSO and mixed with sterile molten potato dextrose agar (PDA, $200 \mathrm{~g}$ potato, $20 \mathrm{~g}$ glucose, $18 \mathrm{~g}$ agar, add water and boil to prepare $1000 \mathrm{~mL}$ solution) medium to obtain a final concentration of 100 and $50 \mu \mathrm{g} / \mathrm{mL}$. DMSO in sterile distilled water was used as the negative control, commercial fungicide hymexazol was selected as a positive control. Place fungi mycelia disks ( $4 \mathrm{~mm}$ diameter) at the center of Petri dishes in a sterile environment and the treatments were incubated in the dark at $25 \pm 1{ }^{\circ} \mathrm{C}$. Each treatment was produced in three replicates. The diameters of the sample colonies were measured, when the colonies in the control experiment covered two-thirds of the culture dishes. Inhibitory percentages of the title compounds in vitro on these fungi were calculated as $I=[(C-T) /(C-4)] \times 100 \%$, where $I$ was the inhibition rate (\%), $\mathrm{C}$ was the diameter $(\mathrm{mm})$ of the fungal colony on the negative control group, and $T$ was the diameter ( $\mathrm{mm}$ ) of the fungal colony on the experimental group.

\section{Results}

\section{Spectral characteristic of title compounds}

The structures of the title compounds were confirmed by ${ }^{1} \mathrm{H}$ NMR, ${ }^{13} \mathrm{C}$ NMR and HRMS data. Here, we take the compound 5a as the example to analyze the molecular structure. In the ${ }^{1} \mathrm{H}$ NMR spectra of the compound 5a (Fig. S1), the broad singlet proton peak was at $12.73 \mathrm{ppm}$ of the amide group and $\left(-\mathrm{NCH}_{2} \mathrm{~N}-,-\mathrm{SCH}_{2} \mathrm{~N}-\right)$ proton of the 1,3,5-thiadiazinethione were about 4.76 ppm and $4.69 \mathrm{ppm}$, respectively. Other $\left(-\mathrm{CH}_{2} \mathrm{C}=\mathrm{O}\right)$ protons were at around $4.02 \mathrm{ppm}$. The ${ }^{13} \mathrm{C}$ NMR peak of the thiophenone group $(-\mathrm{C}=\mathrm{S})$ emerged between 194.03 and $190.48 \mathrm{ppm}$, and carbonyl group $(-\mathrm{C}=\mathrm{O})$ peaks were in the range of $168.65-167.83 \mathrm{ppm}$ (Fig. S2). In addition, the carbon atom peaks were at 2 or 5 position of the 1,3,4-thiadiazole at $159.47-158.02 \mathrm{ppm}$. The HRMS spectra (Fig. S3) exhibited that the compound 5a was peaking at $412.0388\left([\mathrm{M}+\mathrm{H}]^{+}\right)$. For 
more characteristic information about the compounds 5-8, please refer to the Supplemental Information

\section{X-ray crystal structure of compound 8d}

The structure of compound $\mathbf{8 d}$ was further confirmed using the single crystal X-ray analysis. The corresponding crystal structure and crystal packing diagrams were shown in Fig. 4 and Fig. 5, respectively. The hydrogen bonds were given in Table 1. As shown in Fig. 4, the intramolecular hydrogen bond $\mathrm{N}(3)-\mathrm{H}(3) \cdots \mathrm{N}(4)$ formed a new five-membered ring with two other $\mathrm{C}$ atoms. In addition, the intramolecular hydrogen bond $\mathrm{C}(3)-\mathrm{H}(3 \mathrm{~B}) \cdots \mathrm{N}(5)$, together with thiadiazinthion ring constituted a new bridge ring. In the packing diagram of the compound 8d (Fig. 5), the molecules connected each other through intermolecular hydrogen bonds $\mathrm{N}(3)-\mathrm{H}(3) \cdots \mathrm{O}(1)$ and $\mathrm{C}(12)-\mathrm{H}(12) \cdots \mathrm{S}$ (3) (Table 1). Among them, intermolecular hydrogen bond $\mathrm{C}(12)-\mathrm{H}(12) \cdots \mathrm{S}(3)$ connected different molecules to form the molecular chains, while $\mathrm{N}(3)-\mathrm{H}(3) \cdots \mathrm{O}(1)$ connected different chains to form planes, eventually the spatial network was formed. Crystallographic data were deposited with the Cambridge Crystallographic Data Centre. The deposition number was CCDC 1912576.

\section{Antibacterial bioassays of title compounds in vitro}

All the target compounds were tested for the in vitro antibacterial activity against Xoc and Xoo to investigate the biological activity. The preliminary bioassay results demonstrated that all compounds had certain antibacterial activity against $X o c$ and $X o o$ at 100 and $50 \mu \mathrm{g} / \mathrm{mL}$ (Table 2). For example, the inhibitory rates of compounds $5 \mathbf{b}, \mathbf{5 c}, \mathbf{8 c}$ and $8 \mathbf{a}$ against $X o c$ respectively were $22 \%, 22 \%, 18 \%$ and $30 \%$ at $100 \mu \mathrm{g} / \mathrm{mL}$, which are better than that of thiodiazole-copper (18\%). Otherwise, compounds $5 \mathbf{a}-\mathbf{5 d}, \mathbf{6 a}-\mathbf{6 c}, \mathbf{7 a}$ and $\mathbf{8 a}-\mathbf{8 c}$ exhibited certain inhibitory activities against Xoc comparing with the thiodiazole-copper at $50 \mu \mathrm{g} / \mathrm{mL}$. In addition, the title compounds 5a, 6a, $7 \mathbf{a}, 8 \mathbf{a}$ and $8 \mathrm{~b}$ also showed certain activities against $X o o$ at $50 \mu \mathrm{g} / \mathrm{mL}$ and $100 \mu \mathrm{g} / \mathrm{mL}$. Among them, 8a exhibited better antibacterial activities than thiodiazole-copper. As can be seen, compound 8a was the best inhibitor among all the compounds, not only has antibacterial activity against Xoo, but also has a certain inhibitory effect against Xoc.

\section{Antifungal bioassays of title compounds in vitro}

The in vitro inhibitory activity against $R s$ and $F g$ were tested. From the Table 2 it could be found that compounds $5 \mathbf{a}, \mathbf{6 d}, \mathbf{7 d}$ and $\mathbf{8 b}$ possessed of certain activities against both $R s$ and $F g$. At the concentration of $100 \mu \mathrm{g} / \mathrm{mL}$, compound $7 \mathbf{d}$ and $\mathbf{8 b}$ showed comparative activity (46\%,50\%) against $R s$, which was comparable with the commercial drug hymexazol (47\%). Amongst all the compounds, 8 a displayed the best inhibitory activity ( $100 \%$ at $100 \mu \mathrm{g} / \mathrm{mL}$ and $62 \%$ at $50 \mu \mathrm{g} / \mathrm{mL}$ ) 
against $R s$, which was even better than that of hymexazol $(47 \%$ at $100 \mu \mathrm{g} / \mathrm{mL}$ and $37 \%$ at 50 $\mu \mathrm{g} / \mathrm{mL}$ ) (Table 2). Moreover, compound 8a also exhibited good activity (67\% at $100 \mu \mathrm{g} / \mathrm{mL}$ and $43 \%$ at $50 \mu \mathrm{g} / \mathrm{mL}$ ) against $F g$, which was approximate with the activity (66 \% at $100 \mu \mathrm{g} / \mathrm{mL}$ and $43 \%$ at $50 \mu \mathrm{g} / \mathrm{mL}$ ) of hymexazol (Table 2).

Afterwards, the $\mathrm{EC}_{50}$ values of compound $\mathbf{8 a}$ and hymexazol against $R s$ and $F g$ were tested respectively, which were shown in Table $\mathbf{3}$ and Fig. 6. The $\mathrm{EC}_{50}$ value of $\mathbf{8 a}$ against $F g$ was 88.7 $\mu \mathrm{g} / \mathrm{mL}$, which was higher than that of hymexazol $(56.19 \mu \mathrm{g} / \mathrm{mL})$. But the compound 8a showed remarkable activity against $R s$ with an $\mathrm{EC}_{50}$ value of $33.70 \mu \mathrm{g} / \mathrm{mL}$, which was superior to that of hymexazol $(67.10 \mu \mathrm{g} / \mathrm{mL})$.

\section{Discussion}

\section{Synthesis}

The core intermediates 3 were obtained by three steps according to the reported method (Echemendia et al., 2017) with small modification. The 5-amino-1,3,4-thiadiazole-2-thiol reacted with substituted ethyl bromide or substituted benzyl chloride in acetonitrile to form the intermediates 4 with the participation of potassium carbonate. To our disappointment, the reaction time was long and the yield was low (about 40\%). When DMF was used as the solvent, the reaction was completed in $2-8 \mathrm{~h}$ at the same temperature, and the yield was increased to $80 \%$. Optimal conditions for the reaction of intermediates 3 and $\mathbf{4}$ to produce the products 5-8. First, an attempt was made to convert intermediates $\mathbf{3}$ to the corresponding acid chlorides and then reacted with 4. Unfortunately, the reaction failed. Subsequently, different methods, such as the use of 1-(3-dimethylaminopropyl)-3-ethylcarbodiimide (EDCI) hydrochloride/ 1hydroxybenzotriazole $(\mathrm{HOBt}) /$ triethylamine $\left(\mathrm{Et}_{3} \mathrm{~N}\right), \mathrm{EDCI} / 4$-dimethylaminopyridine (DMAP)/ $\mathrm{Et}_{3} \mathrm{~N}$, gave no reaction. However, it was pleasure that the $\mathrm{TBTU} / \mathrm{Et}_{3} \mathrm{~N}$ could make the reaction completed in $2 \mathrm{~h}$ at the room temperature. The result showed that the reaction was carried out well in the condition of $\mathrm{TBTU} / \mathrm{Et}_{3} \mathrm{~N}$, which had many advantages, such as the short reaction time, the reaction mild conditions and the simple and convenient post-treatment. In addition, the yield was $41 \%-79 \%$. It is observed that when $\mathrm{R}^{1}$ was $4-\mathrm{FPh}$, the yield is lower than that of Phenyl, which might be related to the electron-withdrawing group on the benzene ring.

\section{Antimicrobial activity}

From the Table 2, it can be concluded that the changes of the substituents $\mathrm{R}^{1}$ and $\mathrm{R}^{2}$ has a certain effect on the biological activity of the compounds.

For Xoc, it was seen that compounds 5, 8 displayed slightly higher activity than compounds $\mathbf{6}$ and 7 series. This indicated that when the $\mathrm{R}^{1}$ substituent group was substituted with a methyl or phenyl, the corresponding compounds generally exhibited better anti-Xoc effects than those 
compounds bearing a benzyl or 4-fluorophenyl. On the whole, $5 \mathbf{5 a}, \mathbf{5 b}, \mathbf{5 c}, \mathbf{6 a}, \mathbf{6 c}, \mathbf{8 a}$ and $8 \mathbf{c}$ had better activity than the others. Among them, the inhibition rates of $5 \mathbf{a}, \mathbf{6 a}, \mathbf{6 c}$ and $\mathbf{8 c}(17 \%, 17 \%$, $17 \%$ and $18 \%$ ) were approximate with the inhibition rate of commercial bactericide thiodiazolecopper (18\%) at $100 \mu \mathrm{g} / \mathrm{mL}$. This result also applied to the concentration of $50 \mu \mathrm{g} / \mathrm{mL}$. At 100 $\mu \mathrm{g} / \mathrm{mL}$, the activity of compounds $\mathbf{5 b}$ and $\mathbf{5 c}(22 \%$ and $25 \%)$ was slightly higher than that of thiodiazole-copper. This difference was even great at $50 \mu \mathrm{g} / \mathrm{mL}$, the inhibition rates of $\mathbf{5 b}, \mathbf{5 c}$ and thiodiazole-copper were $18 \%, 20 \%$ and $10 \%$, respectively. The activity of $8 \mathbf{a}(30 \%, 100$ $\mu \mathrm{g} / \mathrm{mL} ; 25 \%, 50 \mu \mathrm{g} / \mathrm{mL}$ ) was significantly better than that of thiodiazole-copper.

Bacteria Xoo was more sensitive than Xoc for all the test compounds, including thiodiazolecopper, which was visually displayed in Table $\mathbf{2}$. For Xoo, 5a was the most active compound in compounds $\mathbf{5}$, for which the inhibition rate of $\mathbf{5 a}(30 \%)$ was significantly higher than the others (22\%-24\%). A similar pattern occurs in compounds 6,7 and $\mathbf{8}$, that was $6 \mathbf{6}, 7 \mathbf{a}$ and $8 \mathbf{a}$ were the most active compounds in their respective series. It could be concluded that the substitute $\mathrm{R}^{2}$ played a role in the activity. When the $\mathrm{R}^{1}$ was the same substitute, title compounds bearing a methyl at $\mathrm{R}^{2}$ position exhibited more obvious anti-Xoo effects than the others, which contained benzyl, 4-methyl phenyl or 4-chlorphenyl moiety at $\mathrm{R}^{2}$ position. Among all the target compounds, 6a, 7a and 8a showed higher inhibitory activity than the others, both at $100 \mu \mathrm{g} / \mathrm{mL}$ and $50 \mu \mathrm{g} / \mathrm{mL}$. Compound $8 \mathbf{a}$ was the best inhibitor and showed the inhibition rates of $56 \%$ and $29 \%$ at the concentration of $100 \mu \mathrm{g} / \mathrm{mL}$ and $50 \mu \mathrm{g} / \mathrm{mL}$, respectively, which was compared with the activity of $40 \%$ (at $100 \mu \mathrm{g} / \mathrm{mL}$ ) and $29 \%$ (at $100 \mu \mathrm{g} / \mathrm{mL}$ ) of thiodiazole-copper.

In general, most of the compounds showed a higher antifungal activity towards $R s$ than the $F g$. It can be found that the changes in substituent groups of title compounds greatly influenced their antifungal effects. The structure-activity relationships showed three general rules First, overall, the inhibitory activities of the target compounds against $R s$ were higher than that of $F g$, except for compounds $6 \mathbf{b}$ and $7 \mathbf{c}$. Second, when $\mathrm{R}^{1}$ was $\mathrm{Me}$, that was to say $\mathbf{8 a}-\mathbf{8 d}$, exhibited better antifungal effects than those compounds when $\mathrm{R}^{1}$ was $\mathrm{Ph}(\mathbf{5 a}-\mathbf{5 d}), 4-\mathrm{FPh}(\mathbf{7 a}-\mathbf{7 d})$ or $\mathrm{Bn}(\mathbf{6 a}-\mathbf{6 b})$. Third, when $\mathrm{R}^{2}$ was 4 -Clphenyl, the activities of the corresponding compounds were superior to these compounds with $\mathrm{R}^{2}$ was 4-Mephenyl. For example, the compounds fell into order by inhibitory rate as $\mathbf{5 d}>\mathbf{5 c}, \mathbf{6 d}>\mathbf{6 c}, \mathbf{7 d}>\mathbf{7 c}$ and $\mathbf{8 d}>\mathbf{8 c}$, against both fungi at the concentration of $100 \mu \mathrm{g} / \mathrm{mL}$.

From the antimicrobial activity against $X o c, X o o, R s$ and $F g$ (Table 2 and Table 3), it was indicated that the changes of substituent groups $\mathrm{R}^{1}$ and $\mathrm{R}^{2}$ greatly influenced the antimicrobial activities of the title compounds. In this paper, it was found that when $\mathrm{R}^{1}$ and $\mathrm{R}^{2}$ were the methyl group, the target compound (8a) showed distinct antibacterial and antifungal activities. The compound 8a showed better antimicrobial activities against $X o c$ and $R s$ than the commercial thiodiazole-copper and hymexazol, respectively.The activity results provided a direction for 
further molecular structure optimization of 1,3,5-thiadiazine-2-thione derivatives. The related research is continuing in our laboratory.

\section{Conclusions}

Sixteen novel 1,3,5-thiadiazine-2-thione derivatives containing a 1,3,4-thiadiazole group was designed, synthesized, characterized and screened for the antibacterial effects against $X o c$ and $X o o$ as well as the antifungal effects against $R s$ and $F g$.The antimicrobial bioassays showed that some title compounds displayed valuable antibacterial and antifungal activities. The compound 8a, in which $\mathrm{R}^{1}$ and $\mathrm{R}^{2}$ were the methyl group, was the one of the most prominent activities against all the test microbial. The compound 8a possessed of meaningful antibacterial effects against $X o c$, with the inhibition rates of $30 \%$ at $100 \mu \mathrm{g} / \mathrm{mL}$ and $25 \%$ at $50 \mu \mathrm{g} / \mathrm{mL}$, respectively, which were higher than that of thiodiazole-copper ( $18 \%$ at $100 \mu \mathrm{g} / \mathrm{mL}$ and $10 \%$ at $50 \mu \mathrm{g} / \mathrm{mL})$. In addition, the compound $8 \mathbf{a}$ exhibited better antifungal activity against $R s\left(\mathrm{EC}_{50}=33.70 \mu \mathrm{g} / \mathrm{mL}\right)$ than hymexazol $\left(\mathrm{EC}_{50}=67.10 \mu \mathrm{g} / \mathrm{mL}\right)$. Our research found that the $\mathrm{R}^{1}$ and $\mathrm{R}^{2}$ groups of the title compounds played a vital role in the antibacterial and antifungal activities. However, these studies can provide reference for similar chemical researches in the future. In the future, higher antimicrobial compounds against phytopathogenic microorganisms might be obtained via the further structural modification of 1,3,5-thiadiazine-2-thione derivatives.

\section{References}

\section{Arshad N, Hashim J, Irfanullah Minhas MA, Aslam J, Ashraf T, Hamid SZ, Iqbal T, Javed}

S. 2018. New series of 3,5-disubstituted tetrahydro- $2 H-1,3,5$-thiadiazine thione (THTT) derivatives: synthesis and potent antileishmanial activity. Bioorganic and Medicinal Chemistry Letters 28:3251-3254 DOI 10.1016/j.bmcl.2018.07.045.

\section{Casey JR, Morgan PE, Vullo D, Scozzafava A, Mastrolorenzo A, Supuran C. 2004.}

Carbonic anhydrase inhibitors. Design of selective, membrane-impermeant inhibitors targeting the human tumor-associated isozyme IX. Journal of Medicinal Chemistry 47(9):2337-2347 DOI 10.1021/jm031079w.

Chen HS, Li ZM, Han YF. 2000. Synthesis and fungicidal activity against Rhizoctonia solani of 2-alkyl(Alkylthio)-5-pyrazolyl-1,3,4-oxadiazoles(Thiadiazoles). Journal of Agricultural and Food Chemistry 48(11):5312-5315 DOI 10.1021/jf991065s.

Chen M, Wang XF, Wang SS, Feng YX, Chen F, Yang CL. 2012. Synthesis, characterization and fungicidal activities of novel fluorinated 3,5-disubstituted-4H-1,2,4-triazol-4-amines. Journal of Fluorine Chemistry 135:323-329 DOI 10.1016/j.jfluchem.2011.12.015.

Chen Z, Xu WM, Liu KM, Yang S, Fan HT, Bhadury PS, Hu DY, Zhang YP. 2010. Synthesis and antiviral activity of 5-(4-Chlorophenyl)-1,3,4-thiadiazole sulfonamides. 
Molecules 15(12):9046-9056 DOI 10.3390/molecules15129046.

Coro J, Atherton R, Little S, Wharton H, Yardley V, Alvarez A Jr. Suarez M, Perez R, Rodriguez H. 2006. Alkyl-linked bis-THTT derivatives as potentin vitro trypanocidal agent. Bioorganical and Medicinal Chemistry Letters 16:1312-1315 DOI 10.1016/j.bmcl.2005.11.060.

Coro J, Perez R, Rodriguez H, Suarez M, Vega C, Rolon M, Montero D, Nogal JJ, GomezBarrio A. 2005. Synthesis and antiprotozoan evaluation of new alkyl-linked bis(2thioxo[1,3,5]thiadiazinan-3-yl) carboxylic acids. Bioorganic and Medicinal Chemistry 13:3413-3421 DOI 10.1016/j.bmc.2005.03.009.

Coura, José Rodrigues, Castro S LD. 2002. A critical review on chagas disease chemotherapy. Memórias do Instituto Oswaldo Cruz 97(1):3-24 DOI 10.1590/S0074-02762002000100001.

Cummings SD. 2009. Platinum complexes of terpyridine: Synthesis, structure and reactivity. Coordination Chemistry Reviews 253(3-4):449-478 DOI 10.1016/j.ccr.2008.04.013.

Echemendía R, Fernández O, Coro J, Suárez M, Rivera DG. 2017. A versatile approach to hybrid thiadiazine-based molecules by the Ugi four-component reaction. Tetrahedron Letters 58(18):1784-1787 DOI 10.1016/j.tetlet.2017.03.075.

El-Shorbagi AN, El-Naggar M, Tarazi H, Chaudhary S, AbduAllan H, Hersi F, Omar H. 2018. Bis-(5-substituted-2-thiono1,3,5-thiadiazinan-3-yl) butane as a scaffold of antiproliferative activity, blended by a multicomponent process. Medicinal Chemistry Research 27:1103-1110 DOI 10.1007/s00044-018-2133-9.

Foroumadi A, Kiani Z, Soltani F. 2003. Antituberculosis Agents VIII: Synthesis and in vitro antimycobacterial activity of alkyl $\alpha$-(5-(5-Nitro-2-thienyl)-1,3,4-thiadiazole-2-ylthio)acetates. II Farmaco 58(11):1073-1076 DOI 10.1016/S0014-827X(03)00158-7.

Ji X, Zhong ZM, Chen XL, Xing RG, Liu S, Wang L, Li PC. 2004. Preparation of 1,3,5thiadiazine-2-thione derivatives of chitosan and their potential antioxidant activity in vitro. Bioorganic and Medicinal Chemistry Letters 17:4275-4279 DOI 10.1016/j.bmcl.2007.05.020.

Katiyar D, Tiwari VK, Tripathi RP, Srivastava A, Chaturvedi V, Srivastava R, Srivastava BS. 2003. Synthesis and antimycrobacterial activity of 3,5-disubstituted thiadiazine thiones. Bioorganic and Medicinal Chemistry 11:4369-4375 DOI 10.1016/S0968-0896(03)00480-2.

Khan I, Ali S, Hameed S, Rama NH, Hussain MT, Wadood A, Uddin R, Ul-Haq Z, Khan A, Ali S, Choudhary MI. 2010. Synthesis, antioxidant activities and urease inhibition of some new 1,2,4-triazole and 1,3,4-thiadiazole derivatives. European Journal of Medicinal Chemistry 45(11): 5200-5207 DOI 10.1016/j.ejmech.2010.08.034.

Kumar H, Javed S A, Khan S A, Amir M. 2008. 1,3,4-Oxadiazole/thiadiazole and 1,2,4triazole derivatives of biphenyl-4-yloxy acetic acid: Synthesis and preliminary evaluation of biological properties. European Journal of Medicinal Chemistry 43(12):2688-2698 DOI 
10.1016/j.ejmech.2008.01.039.

Lam WW, Kim JH, Sparks SE, Quistad GB, Casida JE. 1993. Metabolism in rats and mice of the soil fumigants metham, methyl isothiocyanate, and dazomet. Journal of Agricultural and Food Chemistry 41(9):1497-1502 DOI 10.1021/jf00033a027.

Li LX, Jiao J, Wang XB, Chen M, Fu XC, Si WJ, Yang CL. 2018. Synthesis, characterization, and antifungal activity of novel benzo[4,5]imidazo[1,2-d][1,2,4]triazine derivatives. Molecules 23(4):746 DOI 10.3390/molecules23040746.

\section{Li P, Shi L, Yang X, Yang L, Chen XW, Wu F, Shi QC, Xu WM, He M, Hu DY, Song BA.} 2014. Design, synthesis, and antibacterial activity against rice bacterial leaf blight and leaf streak of 2,5-substituted-1,3,4-oxadiazole/thiadiazole sulfone derivative. Bioorganic and Medicinal Chemistry Letters 24(7):1677-1680 DOI 10.1016/j.bmcl.2014.02.060.

Liu WD, Liu JL, Triplett L, Leach JE, Wang GL. 2013. Novel insights into rice innate immunity against bacterial and fungal pathogens. Annual Review of Phytopathology 52(1):213-241 DOI 10.1146/annurev-phyto-102313-045926.

Luo YP, Yang GF. 2007. Discovery of a new insecticide lead by optimizing a target-diverse scaffold: Tetrazolinone derivatives. Bioorganic and Medicinal Chemistry 15(4):1716-1724 DOI 10.1016/j.bmc.2006.12.002.

Mao LG, Jiang HY, Wang QX, Yan DD, Cao AC. 2017. Efficacy of soil fumigation with dazomet for controlling ginger bacterial wilt (Ralstonia solanacearum) in China. Crop Protection 100:111-116 DOI 10.1016/j.cropro.2017.06.013.

Nakamura M, Noda S, Kosugi M, Ishiduka N, Mizukoshi K, Taniguchi M, Nemoto S. 2010. Determination of dithiocarbamates and milneb residues in foods by gas chromatography-mass spectrometry. Food Hygiene Safety Science 51(5):213-219 DOI 10.3358/shokueishi.51.213.

Qian XH, Lee PW, Cao S. 2010. China: Forward to the green pesticides via a basic research program. Journal of Agricultural and Food Chemistry 58(5):2613-2623 DOI 10.1021/jf904098w.

Semreen MH, El-Shorbagi AN, Al-Tel TH, Alsalahat IMM. 2010. Targeting $\gamma$-aminobutyric acid (GABA) carriers to the brain: potential relevance as antiepileptic pro-drugs. Medicinal Chemistry 6:144-149 DOI 10.1093/rheumatology/ket353.

Sheldrick, GM. 1997. SHELXL 97, Program for crystal structure refinement; University of Göttingen: Göttingen, Germany

Siddiqui N, Andalip, Bawa S, Ali R, Afzal O, Akhtar MJ, Azad B, Kumar R. 2011. Antidepressant potential of nitrogen-containing heterocyclic moieties: An updated review. Journal of Pharmacy and Bioallied Sciences 3(2):194-212 DOI 10.4103/0975-7406.80765.

Si WJ, Wang XB, Chen M, Wang MQ, Lu AM, Yang CL. 2019. Design, synthesis, antifungal activity and 3D-QSAR study of novel pyrazole carboxamide and niacinamide derivatives 
containing benzimidazole moiety. New Journal of Chemistry 43: 3000-3010 DOI 10.1039/C8NJ05150J.

Spek A. 2003. Single-crystal structure validation with the program PLATON. Journal of applied crystallography 36:7-13 DOI 10.1107/S0021889802022112.

Vicentini CB, Forlani G, Manfrini M, Romagnoli C, Mares D. 2002. Development of new fungicides against magnaporthe grisea: synthesis and biological activity of pyrazolo[3,4d] $[1,3]$ thiazine, pyrazolo[1,5-c][1,3,5]thiadiazine, and pyrazolo[3,4-d]pyrimidine derivatives. Journal of Agricultural and Food Chemistry 50:4839-4845 DOI 10.1021/jf0202436.

Vicentini CB, Guccione S, Giurato L, Ciaccio R, Mares D, Forlani G. 2005. Pyrazole derivatives as photosynthetic electron transport inhibitors: new leads and structure-activity relationship. Journal of Agricultural and Food Chemistry 53:3848-3855 DOI $10.1021 / \mathrm{jf0500029.}$

Wan JL, Gan YY, Hu WN, Meng J, Tian K, LiXQ, Wu SQ, XuY,Ouyang GP, Wang ZC. 2018. Design, Synthesis and Anti-bacterial Evaluation of Novel 1,3,4-Thiadiazole Derivatives Bearing a Semicarbazone Moiety. Phosphorus, Sulfur, and Silicon and the Related Elements 193(7):443-450 DOI 10.1080/10426507.2018.1436546.

Wang X, Li P, Li Z, Yin J, He M, Xue W, Chen ZW, Song BA. 2013. Synthesis and bioactivity evaluation of novel arylimines containing a 3-aminoethyl-2-( $p$-trifluoromethoxy) anilino-4(3H)-quinazolinone moiety. Journal of Agricultural and Food Chemistry 61(40):9575-9582 DOI 10.1021/jf403193q.

Wang X, Dai ZC, Chen YF, Cao LL, Yan W, Li SK, Wang JX, Zhang ZG, Ye YH. 2017. Synthesis of 1,2,3-triazole hydrazide derivatives exhibiting anti-phytopathogenic activity. European Journal of Medicinal Chemistry 126:171-182 DOI 10.1016/j.ejmech.2016.10.006.

Wang XB, Fu XC, Yan JH, Wang A, Wang MQ, Chen M, Yang CL, Song YM. 2018. Design and synthesis of novel 2-(6-thioxo-1,3,5-thiadiazinan-3-yl)- $N$ '-phenylacethydrazide derivatives as potential fungicides. Molecular Diversity 1-11 DOI 10.1007/s11030-018-98917.

Wilson RA, Talbot NJ. 2009. Fungal physiology-a future perspective. Microbiology 155(12):3810-3815 DOI 10.1099/mic.0.035436-0.

Xu WM, Yang S, Bhadury P, He J, He M, Gao LL, Hu DY, Song BA. 2011. Synthesis and bioactivity of novel sulfone derivatives containing 2,4-dichlorophenyl substituted 1,3,4oxadiazole/thiadiazole moiety as chitinase inhibitors. Pesticide Biochemistry and Physiology 101(1):6-15 DOI 10.1016/j.pestbp.2011.05.006.

Xu WM, Han FF, He M, Hu DY, He J, Yang S, Song BA. 2012. Inhibition of tobacco bacterial wilt with sulfone derivatives containing an 1,3,4-oxadiazole moiety. Journal of Agricultural Food Chemistry 60:1036-1041 DOI 10.1021/jf203772d. 
573 Zhong XM, Wang XB, Chen LJ, Ruan XH, Li Q, Zhang JP, Chen Z, Xue W. 2017.

574 Synthesis and biological activity of myricetin derivatives containing 1,3,4-thiadiazole scaffold.

575

Chemistry Central Journal 11(1):106 DOI 10.1186/s13065-017-0336-7.

576 
Figure 1

Bioactive compounds containing a 1,3,5-thiadiazine-2-thione or 1,3,4-thiadiazole fragment.

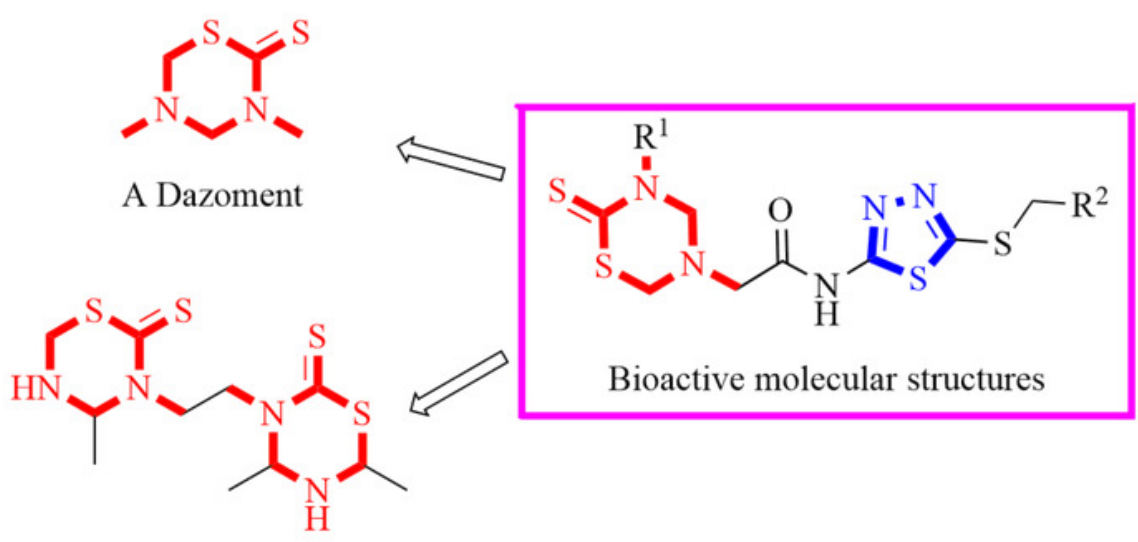

B Milneb

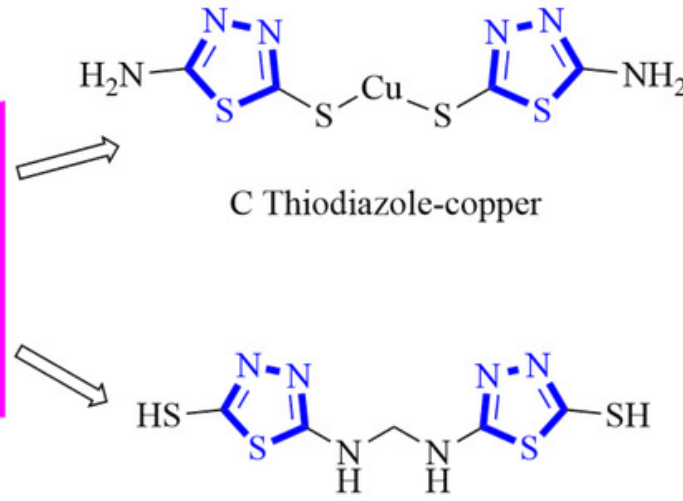

D Bismerthiazol 
Figure 2

Synthetic route to title compounds 5-8.
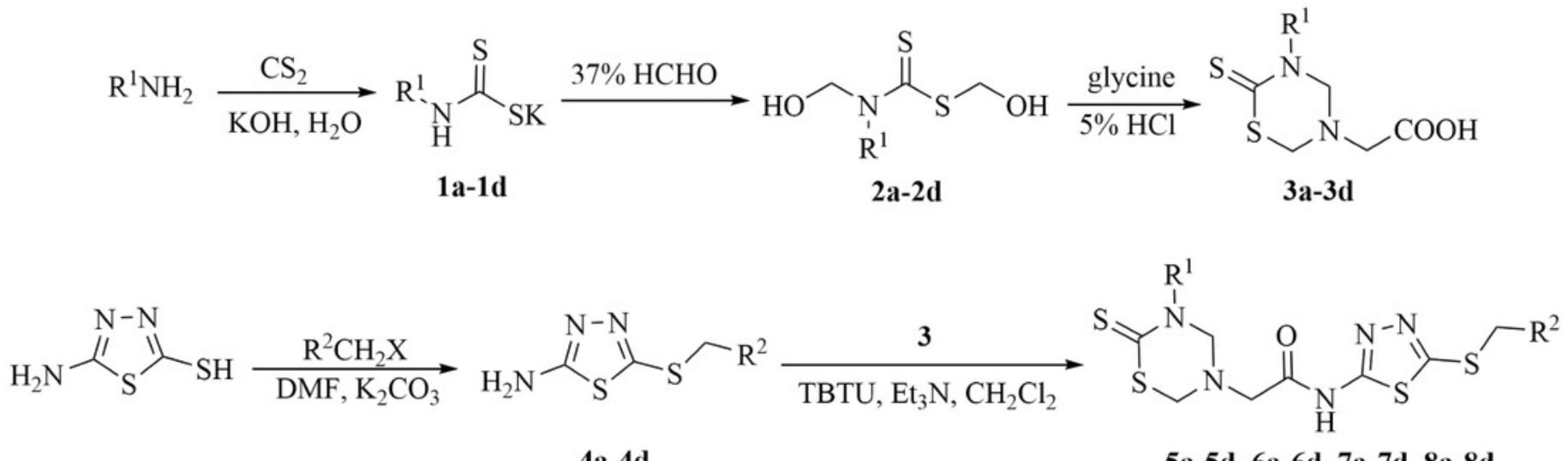
1a, 2a, 3a, 5a-5d: $\mathrm{R}^{1}=\mathrm{Ph}$
1b, 2b, 3b, 6a-6d: $R^{1}=B n$
1c, 2c, 3c, 7a-7d: $\mathrm{R}^{1}=4-\mathrm{FPh}$
1d, 2d, 3d, 8a-8d: $R^{1}=M e$

4a, 5a, 6a, 7a, 8a: $\mathrm{R}^{2}=\mathrm{Me}$

4b, 5b, 6b, 7b, 8b: $\mathrm{R}^{2}=\mathrm{Ph}$

$4 c, 5 c, 6 c, 7 c, 8 c: \mathrm{R}^{2}=4-\mathrm{MePh}$

4d, 5d, 6d, 7d, 8d: $\mathrm{R}^{2}=4-\mathrm{ClPh}$ 
Figure 3

Design strategies for title compounds.<smiles>CN1CN(CC(=O)NN[Al])CSC1=S</smiles>

Our previous work

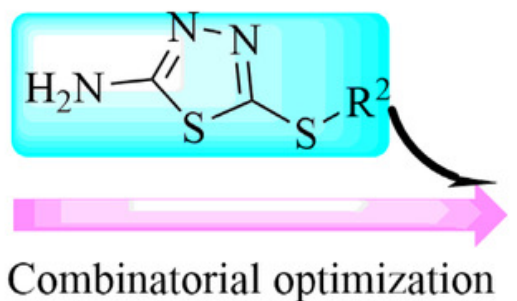

Combinatorial optimization

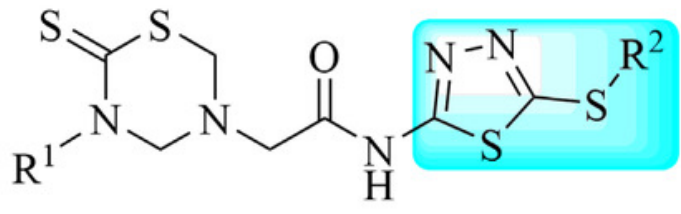

Our present work 
Figure 4

The molecular ellipsoid of compound 8d.

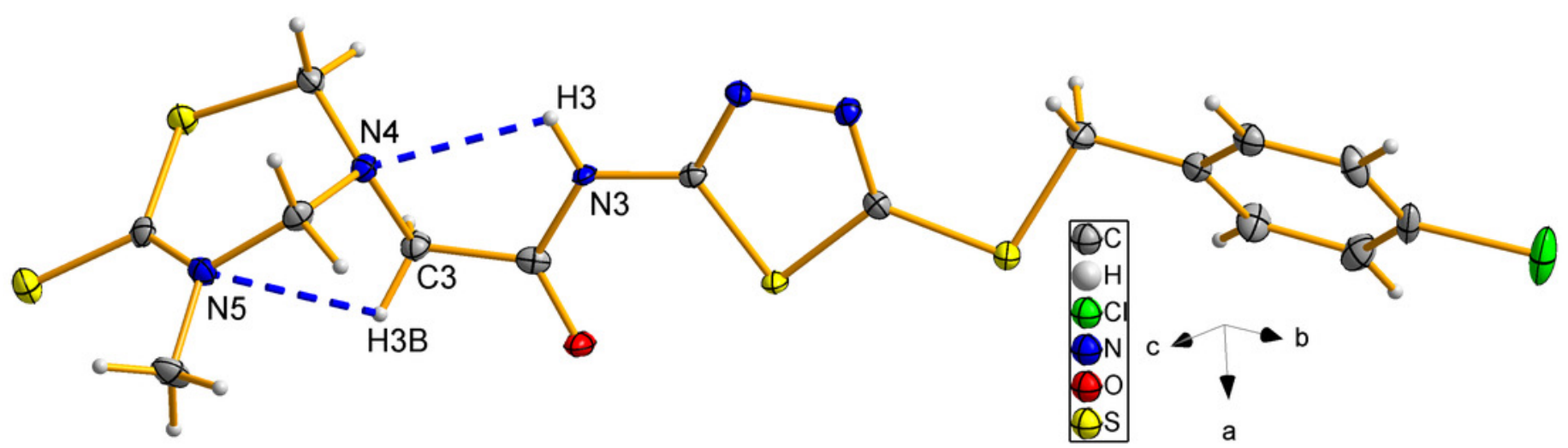


Figure 5

Crystal packing diagram of compound 8d

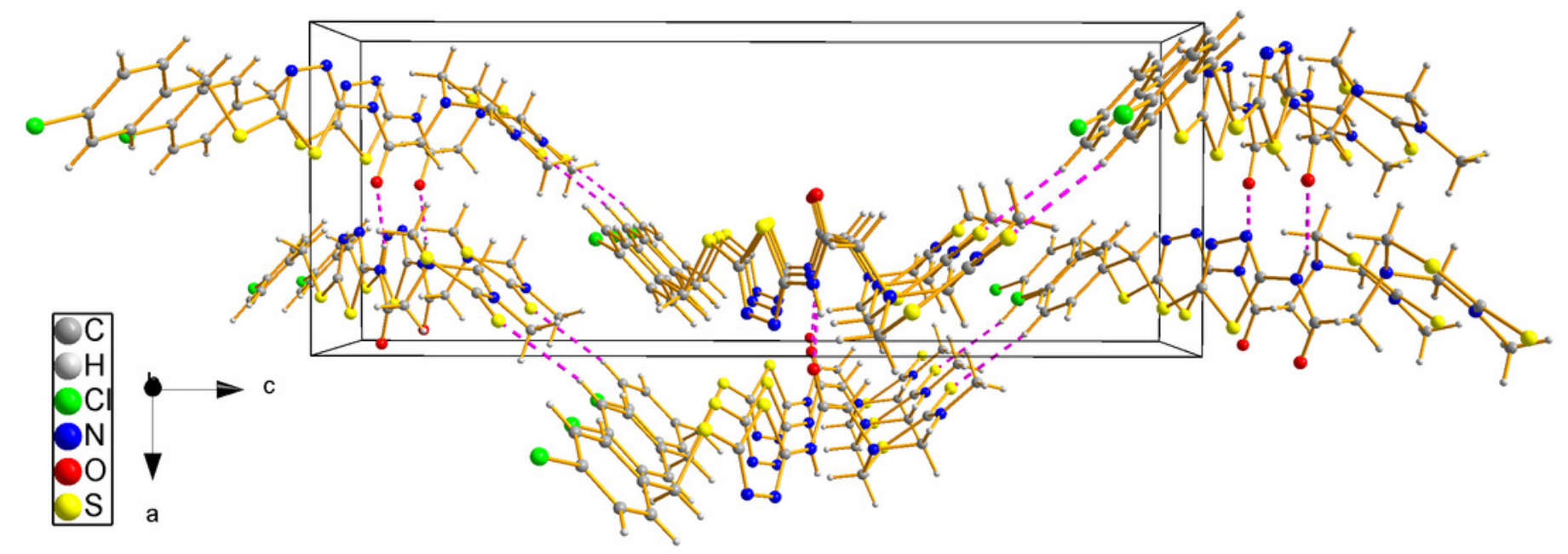




\section{Table 1 (on next page)}

Hydrogen bond distances $(\AA)$ and angles $\left({ }^{\circ}\right)$ of compound $8 d$. 
1

\begin{tabular}{lllll}
\hline $\mathrm{D}-\mathrm{H} \cdots \mathrm{A}$ & $d(\mathrm{D}-\mathrm{H})$ & $d(\mathrm{H} \cdots \mathrm{A})$ & $d(\mathrm{D} \cdots \mathrm{A})$ & $\angle(\mathrm{DHA})$ \\
\hline $\mathrm{N}(3)-\mathrm{H}(3) \cdots \mathrm{N}(4)$ & $0.84(4)$ & $2.41(4)$ & $2.759(4)$ & $106(3)$ \\
$\mathrm{C}(3)-\mathrm{H}(3 \mathrm{~B}) \cdots \mathrm{N}(5)$ & 0.9700 & 2.6100 & $3.008(5)$ & 105.00 \\
$\mathrm{~N}(3)-\mathrm{H}(3) \cdots \mathrm{O}(1)^{\mathrm{a}}$ & $0.84(4)$ & $2.00(4)$ & $2.809(4)$ & $160(4)$ \\
$\mathrm{C}(12)-\mathrm{H}(12) \cdots \mathrm{S}(3)^{\mathrm{b}}$ & 0.9300 & 2.8600 & $3.769(4)$ & 165.00 \\
\hline
\end{tabular}

2 Notes: ${ }^{a}$ Symmetry code: $-1 / 2+x, 1 / 2-y, z$; ${ }^{~}$ Symmetry code: $2-x, 1-y,-1 / 2+z$.

3 
Table 2 (on next page)

Inhibition rates of title compounds against phytopathogenic microorganisms ${ }^{a}$. 


\begin{tabular}{|c|c|c|c|c|c|c|c|c|c|c|}
\hline \multirow{2}{*}{ Compd } & \multirow{2}{*}{$\mathrm{R}^{1}$} & \multirow{2}{*}{$\mathrm{R}^{2}$} & \multicolumn{2}{|l|}{$X o c$} & \multicolumn{2}{|l|}{ Xoo } & \multicolumn{2}{|l|}{ Rs } & \multicolumn{2}{|l|}{$\mathrm{Fg}$} \\
\hline & & & $100 \mu \mathrm{g} / \mathrm{mL}$ & $50 \mu \mathrm{g} / \mathrm{mL}$ & $100 \mu \mathrm{g} / \mathrm{mL}$ & $50 \mu \mathrm{g} / \mathrm{mL}$ & $100 \mu \mathrm{g} / \mathrm{mL}$ & $50 \mu \mathrm{g} / \mathrm{mL}$ & $100 \mu \mathrm{g} / \mathrm{mL}$ & $50 \mu \mathrm{g} / \mathrm{mL}$ \\
\hline $5 \mathbf{a}$ & $\mathrm{Ph}$ & $\mathrm{Me}$ & $17 \pm 0.47$ & $14 \pm 3.08$ & $30 \pm 2.02$ & $17 \pm 1.66$ & $35 \pm 3.68$ & $10 \pm 2.24$ & $34 \pm 4.10$ & $18 \pm 3.05$ \\
\hline $5 b$ & $\mathrm{Ph}$ & $\mathrm{Ph}$ & $22 \pm 1.41$ & $18 \pm 4.13$ & $24 \pm 4.10$ & $15 \pm 3.48$ & $14 \pm 1.76$ & $6 \pm 4.65$ & $15 \pm 3.35$ & $7 \pm 3.55$ \\
\hline $5 c$ & $\mathrm{Ph}$ & 4-MePh & $25 \pm 3.54$ & $20 \pm 1.71$ & $22 \pm 0.11$ & $14 \pm 2.55$ & $21 \pm 1.94$ & $5 \pm 1.72$ & $17 \pm 3.69$ & $6 \pm 1.76$ \\
\hline $5 d$ & $\mathrm{Ph}$ & 4-ClPh & $13 \pm 2.15$ & $10 \pm 2.02$ & $24 \pm 0.34$ & $10 \pm 0.10$ & $27 \pm 2.69$ & $15 \pm 3.40$ & $20 \pm 4.18$ & $7 \pm 2.29$ \\
\hline $6 a$ & $\mathrm{Bn}$ & $\mathrm{Me}$ & $17 \pm 4.18$ & $10 \pm 1.15$ & $38 \pm 1.06$ & $26 \pm 0.55$ & $23 \pm 1.69$ & $13 \pm 2.74$ & $23 \pm 1.39$ & $12 \pm 2.96$ \\
\hline $6 \mathbf{b}$ & $\mathrm{Bn}$ & $\mathrm{Ph}$ & $14 \pm 3.31$ & $11 \pm 2.11$ & $29 \pm 4.18$ & $19 \pm 0.69$ & $18 \pm 1.47$ & $14 \pm 3.27$ & $26 \pm 4.44$ & $18 \pm 2.61$ \\
\hline $6 c$ & $\mathrm{Bn}$ & 4-MePh & $17 \pm 0.92$ & $11 \pm 1.52$ & $20 \pm 4.11$ & $13 \pm 0.43$ & $29 \pm 2.44$ & $22 \pm 4.10$ & $26 \pm 4.71$ & $12 \pm 1.88$ \\
\hline $6 d$ & $\mathrm{Bn}$ & 4-ClPh & $9 \pm 4.74$ & $5 \pm 1.13$ & $25 \pm 0.43$ & $16 \pm 0.55$ & $30 \pm 2.20$ & $15 \pm 1.83$ & $32 \pm 3.41$ & $15 \pm 2.79$ \\
\hline $7 a$ & 4-FPh & $\mathrm{Me}$ & $14 \pm 0.01$ & $12 \pm 1.35$ & $35 \pm 0.91$ & $25 \pm 0.02$ & $21 \pm 1.69$ & $10 \pm 2.24$ & $15 \pm 3.66$ & $11 \pm 2.69$ \\
\hline $7 b$ & 4-FPh & $\mathrm{Ph}$ & $13 \pm 3.42$ & $6 \pm 3.77$ & $26 \pm 2.97$ & $20 \pm 0.70$ & $22 \pm 1.67$ & $9 \pm 1.70$ & $19 \pm 3.51$ & $6 \pm 2.43$ \\
\hline $7 \mathrm{c}$ & 4-FPh & 4-MePh & $15 \pm 3.85$ & $9 \pm 2.10$ & $24 \pm 0.36$ & $17 \pm 4.63$ & $8 \pm 1.67$ & 0 & $14 \pm 3.47$ & $6 \pm 3.03$ \\
\hline $7 d$ & 4-FPh & 4-ClPh & $10 \pm 7.54$ & $5 \pm 4.44$ & $25 \pm 0.91$ & $13 \pm 2.30$ & $46 \pm 2.44$ & $28 \pm 4.10$ & $14 \pm 1.75$ & $7 \pm 2.04$ \\
\hline $8 a$ & $\mathrm{Me}$ & $\mathrm{Me}$ & $30 \pm 2.58$ & $25 \pm 5.87$ & $56 \pm 2.02$ & $29 \pm 2.03$ & 100 & $62 \pm 6.37$ & $67 \pm 7.14$ & $43 \pm 5.05$ \\
\hline $8 b$ & $\mathrm{Me}$ & $\mathrm{Ph}$ & $16 \pm 4.13$ & $12 \pm 3.61$ & $32 \pm 0.36$ & $26 \pm 4.63$ & $50 \pm 5.89$ & $36 \pm 3.00$ & $30 \pm 5.80$ & $16 \pm 3.39$ \\
\hline $8 \mathrm{c}$ & $\mathrm{Me}$ & 4-MePh & $18 \pm 4.68$ & $15 \pm 4.40$ & $20 \pm 5.12$ & $12 \pm 3.49$ & $19 \pm 4.76$ & $13 \pm 2.26$ & $20 \pm 1.47$ & $9 \pm 2.59$ \\
\hline $8 d$ & $\mathrm{Me}$ & 4-ClPh & $13 \pm 2.80$ & $10 \pm 4.00$ & $25 \pm 4.12$ & $19 \pm 4.05$ & $22 \pm 6.15$ & $7 \pm 2.59$ & $21 \pm 1.52$ & $12 \pm 3.49$ \\
\hline $\mathrm{TC}^{\mathrm{b}}$ & - & - & $18 \pm 4.77$ & $10 \pm 2.79$ & $40 \pm 1.02$ & $29 \pm 4.43$ & - & - & - & - \\
\hline $\mathrm{HY}^{\mathrm{b}}$ & - & - & - & - & - & - & $47 \pm 6.94$ & $37 \pm 7.70$ & $66 \pm 4.09$ & $43 \pm 1.89$ \\
\hline
\end{tabular}

2 Notes: ${ }^{a}$ Average of three replicates; ${ }^{\mathrm{b}}$ A commercial agricultural bacterial thiodiazole-copper and hymexazol were used for comparison of antibacterial activities. 


\section{Figure 6}

Anti-Rs effects of the bioactive compounds $8 a$ and hymexazol.

(A) $8 \mathbf{a}$ at $50 \mu \mathrm{g} / \mathrm{mL}$, (B) $8 \mathbf{a}$ at $25 \mu \mathrm{g} / \mathrm{mL}$, (C) $8 \mathbf{a}$ at $12.5 \mu \mathrm{g} / \mathrm{mL}$, (D) $8 \mathbf{a}$ at $6.25 \mu \mathrm{g} / \mathrm{mL}$, (E) $8 \mathbf{a}$ at

$3.125 \mu \mathrm{g} / \mathrm{mL}$, (F) hymexazol at $50 \mu \mathrm{g} / \mathrm{mL},(\mathrm{G})$ hymexazol at $25 \mu \mathrm{g} / \mathrm{mL},(\mathrm{H})$ hymexazol at 12.5 $\mu \mathrm{g} / \mathrm{mL}$, (I) hymexazol at $6.25 \mu \mathrm{g} / \mathrm{mL}$ and (J) hymexazol at $3.125 \mu \mathrm{g} / \mathrm{mL}$.

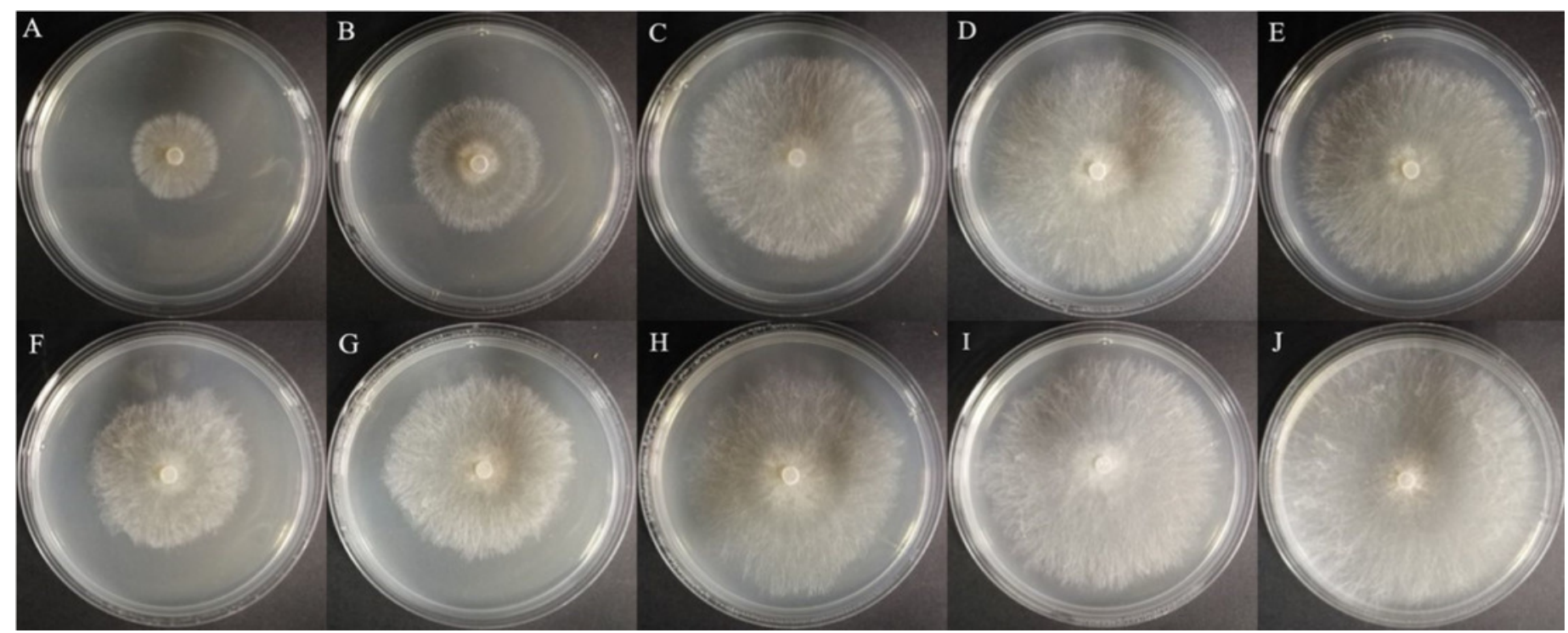


Table 3 (on next page)

$\mathrm{EC}_{50}$ values of the title compound 8a against $R s$ and $\mathrm{Fg}$. 
1

\begin{tabular}{lllll}
\hline Compd & Strains & Regression equation & $\mathrm{r}$ & $\mathrm{EC}_{50}(\mu \mathrm{g} / \mathrm{mL})^{\mathrm{a}}$ \\
\hline $\mathbf{8 a}$ & $R s$ & $\mathrm{y}=2.1218 \mathrm{x}+1.7578$ & 0.9925 & $33.70 \pm 0.24$ \\
$\mathbf{8 a}$ & $F g$ & $\mathrm{y}=1.2648 \mathrm{x}+2.5363$ & 0.9654 & $88.70 \pm 0.49$ \\
Hymexazol $^{\mathrm{b}}$ & $R s$ & $\mathrm{y}=1.5892 \mathrm{x}+2.0968$ & 0.9869 & $67.10 \pm 0.24$ \\
Hymexazol $^{\text {Hy }}$ & $F g$ & $\mathrm{y}=1.8967 \mathrm{x}+1.6815$ & 0.9986 & $56.19 \pm 1.68$ \\
\hline
\end{tabular}

2 Notes: ${ }^{a}$ Average of three replicates; ${ }^{b}$ Hymexazol, the agricultural fungicide, was used for the comparison of antifungal effects. 3 